\title{
Synthesis, Biological Evaluation, and QSAR Studies of 3-lodochromone Derivatives as Potential Fungicides
}

\author{
Parshant Kaushik*, Najam A. Shakil and Virendra S. Rana \\ Division of Agricultural Chemicals, ICAR-Indian Agricultural Research Institute, New Delhi, India
}

OPEN ACCESS

Edited by:

Simone Brogi,

University of Pisa, Italy

Reviewed by:

Rajeev K. Singla,

Sichuan University, China

Andrei I. Khlebnikov,

Tomsk Polytechnic University,

Russia

*Correspondence:

Parshant Kaushik

parshantagrico@gmail.com

Specialty section: This article was submitted to Medicinal and Pharmaceutical

Chemistry,

a section of the journal

Frontiers in Chemistry

Received: 11 December 2020

Accepted: 17 February 2021

Published: 30 April 2021

Citation:

Kaushik $P$, Shakil NA and Rana VS

(2021) Synthesis, Biological

Evaluation, and QSAR Studies of 3-

lodochromone Derivatives as

Potential Fungicides.

Front. Chem. 9:636882.

doi: 10.3389/fchem.2021.636882
Despite the emergence of novel biotechnological and biological solutions, agrochemicals continue to play an important role in crop protection. Fungicide resistance is becoming a major problem; numerous cases of fungicide resistance have occurred worldwide in the last decade, resulting in the loss of several fungicides. The discovery of new molecules has therefore assumed critical importance in crop protection. In our quest for biologically active molecules, we herein report the synthesis of a series of twenty-one 3-lodochromone derivatives $(4 \mathrm{a}-4 \mathrm{u})$, in a two-step process by condensation of 2-hydroxyacetophenone derivatives $(2 \mathrm{a}-2 \mathrm{U})$ with $\mathrm{N}, \mathrm{N}$-dimethylformamidedimethylacetal yielding enaminones (3a-3u), followed by cyclization with iodine to corresponding 3-iodochromones. Characterization of these compounds was done by $I R,{ }^{1} \mathrm{H} N M R,{ }^{13} \mathrm{C} N M R$, and LCHRMS techniques. All synthesized compounds were screened for their fungicidal activity against Sclerotium rolfsii. Among these 6,8-Dichloro-3-iodochromone $\mathbf{4 r}$ was found to be most active $\left(\mathrm{ED}_{50}=8.43 \mathrm{mg} \mathrm{L}^{-1}\right)$. 2D-Quantitative Structural Activity Relationship (2DQSAR) analysis was also performed by generating three different models viz., Multiple Linear Regression (MLR, Model 1), Principal Component Regression (PCR, Model 2), and Partial Least Squares (PLS, Model 3). Predictive power and statistical significance of these models were assessed with external and internal validation and leave one-out crossvalidation was used for verification. In QSAR study, MLR (Model 1) was found to be best having correlation coefficient $\left(r^{2}\right) 0.943$, cross-validated correlation coefficient $\left(q^{2}\right) 0.911$ and $r^{2}$ pred 0.837. It was observed that DeltaEpsilonC, T_2_Cl_6, T_2_F_6, T_T_F_3, and ZCompDipole are the major descriptors which influence the fungicidal activity of 3lodochromone derivatives. The physicochemical parameters were estimated by the VLifeMDS 4.6 software. The QSAR study results will be helpful for structure optimization to improve the activity.

Keywords: 3-iodochromone, fungicidal activity, sclerotium rolfsii, QSAR, multiple linear regression, principle component analysis, partial least square

\section{INTRODUCTION}

The growth of human civilization has been closely related to crop production, and plant diseases have been a concern for human being perhaps since plants were cultivated more than 10,000 years ago. As a consequence of plant diseases, world agriculture faces an estimated loss of $18 \%$ annually amounting to approximately 1,300 billion INR (Oerke, 2006). Sclerotium rolfsii Sacc. is a devastating soilborne fungus that infects more than five hundred agricultural and horticultural plant species around the 
world, causing root rot, stem rot, collar rot, willow, and foot rot diseases (Aycock, 1966; Punja, 1985). Crucifers, cucurbits, and legumes are its most common hosts. The fungus is of considerable economic significance because it causes 10-100 percent crop loss in different crops. Due to the formation of excessive sclerotia it may persist in soil for several years (Punja, 1985). Chemical crop protection measures continue to play an important role in agribusiness in spite of the emergence of novel biotechnological and biological solutions. Resistance to fungicides is becoming a major problem generating disease control problems in many crops. In the last decade, numerous cases of fungicide resistance have occurred worldwide, leading to loss of several fungicides (Hollomon, 2015). Therefore, the discovery of new molecules has assumed critical importance to combat the fungal infections.

Chromone is a group of naturally occurring compounds, reported, mainly in plants. The chromone moiety is a pharmacophore in a large number of natural and synthetic bioactive molecules. The chromone scaffold is present in plant's secondary metabolites: flavones and isoflavones. Chromones are reported to have anti-tumor, antiinflammatory and anti-fungal activities, and inhibitory activities, toward, phosphatases, kinases, cyclooxygenases, aromatases, acetylcholinesterases, and monoamine oxidases (Gasparova et al., 1997; Gaspar et al., 2011, Gaspar et al., 2014).

Our group has been actively involved in developing new crop protection products (Kaushik et al., 2019; Yadav et al., 2019). In our quest for biologically active molecules, we herein reported the synthesis of a series of iodochromones and their evaluation against S. rolfsii. A QSAR study was carried out with the objective to find the molecular properties which affect the fungicidal activity.

\section{EXPERIMENTAL}

\section{Chemicals and Instruments}

Chemicals were purchased from industrial manufacturers and, unless otherwise specified, were used without any further purification. Precoated Merck-silica gel $60 \mathrm{~F}_{254}$ plates were used for thin layer chromatography (TLC); UV cabinet was used to detect developed plates. Column chromatography was performed with 100-200 mesh silica gels. Melting points were recorded by Buchi M-560 instrument and were uncorrected. The IR spectroscopy was done with PerkinElmer 2000 FT-IR spectrometer; $\mathrm{KBr}$ disc were used for samples preparation. The ${ }^{1} \mathrm{H}$ NMR and ${ }^{13} \mathrm{C}$ spectra were recorded on a Jeol alpha-400 and at $100.6 \mathrm{MHz}$, respectively, using TMS as an internal standard. The chemical shift values were on $\delta$ scale and the coupling constants (J) were in Hz. Signals from $\mathrm{OH}$ groups in ${ }^{1} \mathrm{HNMR}$ spectra were verified by removing them by shaking in $\mathrm{D}_{2} \mathrm{O}$.

High Resolution Mass Spectrometry (HRMS) was performed by AB SCIEX Triple TOFTM 5600+ equipped with Turboion Spray (TIS), SCIEX ExionLC, and PDA detector. Compounds were separated through C-18 column $(2.7 \mu \mathrm{m}, 4.6 \times 100 \mathrm{~mm})$ by eluting with methanol and water $(98: 2, \mathrm{v} / \mathrm{v})$ at $0.3 \mathrm{ml} / \mathrm{min}$ at $40^{\circ} \mathrm{C}$.

$\mathrm{ED}_{50}$ values were estimated with the SPSS statistical package. The whole computational work was carried out by using
VLifeMDS QSAR plus 4.6 software using the Lenovo PC having window 8.1 operating system and Intel (R) Celeron (R) processor.

\section{Synthesis}

\section{Synthesis of Substituted 2-Hydroxyacetophenones (2a-2n)}

2-hydroxyacetophenone and bromoalkanes or iodoalkanes of different chain lengths were taken in a molar ratio of $1: 1.2$ and stirred continuously for $6 \mathrm{~h}$ at $60^{\circ} \mathrm{C}$ in the presence of $\mathrm{K}_{2} \mathrm{CO}_{3}$ and acetone. Reaction was supervised by thin layer chromatography (TLC) with ethyl acetate: hexane (3:7).

\section{General Method for Synthesis of Substituted 3-Dimethylamino-1-(2-hydroxyphenyl)propenones (3a-3u)}

A mixture of substituted 2-hydroxyacetophenones (2a-2u) $(1.2 \mathrm{mmol})$ and $N, N$-dimethylformamidedimethylacetal (2.4 mmol, 2 molequiv) was heated at $90^{\circ} \mathrm{C}$ overnight and allowed to cool. The reaction was worked up by removing solvent using vacuum evaporation on Heidolph rotary evaporator Hei-VAP, and the pure product were obtained by column chromatography with hexane: ethyl acetate (85:15). Compounds 3f, 3g, 3h, 3i, 3j, 3k, 31, 3m, 3n, and $\mathbf{3 o}$ were reported for the first time in the literature.

\section{Spectral Analysis of Synthesized 3-Dimethylamino-1-(2-hydroxyphenyl) propenones (3a-3u)}

3-Dimethylamino-1-(2-hydroxyphenyl)propenone (3a)

It was obtained as a brown solid in $82 \%$ yield; m.p.: $127-128^{\circ} \mathrm{C}, \mathrm{R}_{\mathrm{f}}$ : 0.42 (ethyl acetate: hexane, 3:7). IR $\left(\mathrm{cm}^{-1}\right)$ : 3,710 (O-H stretch), 2,949 (aliphatic C-H), 1,635 (C=O), 1,565 (C=C stretch), 1,455 (C-H bending of $\mathrm{CH}_{2}$ ), and 1,264 (C-O). ${ }^{1} \mathrm{H}$ NMR $(400 \mathrm{MHz}$, $\left.\mathrm{CDCl}_{3}\right): \delta 2.87\left(3 \mathrm{H}, \mathrm{s}, \mathrm{N}-\mathrm{CH}_{3}\right), 3.12\left(3 \mathrm{H}, \mathrm{s}, \mathrm{N}-\mathrm{CH}_{3}\right), 5.73(1 \mathrm{H}, \mathrm{d}, \mathrm{J}$ $=12, \mathrm{H}-3), 6.62-6.73(4 \mathrm{H}, \mathrm{m}, \mathrm{ArH})$, and $7.88(1 \mathrm{H}, \mathrm{d}, \mathrm{J}=12.4, \mathrm{H}-$ 2). ${ }^{13} \mathrm{C}$ NMR (100.6 MHz, $\left.\mathrm{CDCl}_{3}\right): 190.31(\mathrm{C}-1), 162.10\left(\mathrm{C}-2^{\prime}\right)$, 154.05 (C-3), 133.97 (C-4"), $130.08\left(\mathrm{C}-6^{\prime \prime}\right), 122.31\left(\mathrm{C}-1^{\prime \prime}\right)$, 121.42.84 (C-5"), $116.21\left(\mathrm{C}-3^{\prime \prime}\right)$, and 89.75 (C-2). HR-MS for $\mathrm{C}_{11} \mathrm{H}_{13} \mathrm{NO}_{2}[\mathrm{M}+\mathrm{H}]^{+} \mathrm{m} / z$ : Calcd 192.1091; Observed 192.1087. The major mass fragments observed were $\mathrm{C}_{7} \mathrm{H}_{5} \mathrm{O}_{3}{ }^{+}$(137), $\mathrm{C}_{5} \mathrm{H}_{8} \mathrm{O}^{+}(98)$, and $\mathrm{C}_{4} \mathrm{H}_{10} \mathrm{~N}^{+}(72)$.

\section{3-Dimethylamino-1-(2-hydroxy-4-methoxyphenyl) propenone (3b)}

It was obtained as bright light yellow solid in $87 \%$ yield; m.p.: $137-140^{\circ} \mathrm{C}, \mathrm{R}_{\mathrm{f}}: 0.42$ (ethyl acetate: hexane, 3:7). IR $\left(\mathrm{cm}^{-1}\right): 3,711$ (O-H stretch), 2,953 (aliphatic C-H), 1,630 (C=O), 1,561 ( $=\mathrm{C}$ stretch), 1,458 (C-H bending of $\left.\mathrm{CH}_{2}\right)$, and 1,266 (C-O). ${ }^{1} \mathrm{HNMR}$ $\left(400 \mathrm{MHz}, \mathrm{CDCl}_{3}\right): \delta 2.95\left(3 \mathrm{H}, \mathrm{s}, \mathrm{N}-\mathrm{CH}_{3}\right), 3.13\left(3 \mathrm{H}, \mathrm{s}, \mathrm{N}-\mathrm{CH}_{3}\right)$, $3.98\left(2 \mathrm{H}, \mathrm{t}, \mathrm{J}=6.8, \mathrm{H}_{-1}^{\prime}\right), 5.67(1 \mathrm{H}, \mathrm{d}, \mathrm{J}=12, \mathrm{H}-2), 6.33-6.37(2 \mathrm{H}$, $\mathrm{m}, \mathrm{H}-5^{\prime}$ and $\left.\mathrm{H}-3^{\prime}\right), 7.58\left(1 \mathrm{H}, \mathrm{d}, \mathrm{J}=8.8 \mathrm{H}-6^{\prime \prime}\right)$, and $7.85(1 \mathrm{H}, \mathrm{d}, \mathrm{J}=$ 12.4, H-3). ${ }^{13} \mathrm{C}$ NMR (100.6 MHz, $\left.\mathrm{CDCl}_{3}\right): 190.81$ (C-1), 165.53 $\left({ }^{4} \mathrm{C}^{\prime \prime}\right), 164.06\left({ }^{2} \mathrm{C}^{\prime \prime}\right), 154.05(\mathrm{C}-3), 129.79\left(\mathrm{C}-6^{\prime \prime}\right), 113.72\left(\mathrm{C}-1^{\prime \prime}\right)$, $106.84\left(\mathrm{C}-5^{\prime}\right), 101.53\left(\mathrm{C}-3^{\prime \prime}\right)$, and $89.75(\mathrm{C}-2)$. HR-MS for $\mathrm{C}_{12} \mathrm{H}_{15} \mathrm{NO}_{3}[\mathrm{M}+\mathrm{H}]^{+} m / z$ : Calcd 222.1124; Observed 
222.1118. The major mass fragments observed were $\mathrm{C}_{8} \mathrm{H}_{7} \mathrm{O}_{3}{ }^{+}(151), \mathrm{C}_{7} \mathrm{H}_{5} \mathrm{O}_{3}{ }^{+}(137), \mathrm{C}_{5} \mathrm{H}_{8} \mathrm{NO}^{+}(98)$, and $\mathrm{C}_{4} \mathrm{H}_{10} \mathrm{~N}^{+}(72)$.

\section{3-Dimethylamino-1-(2-hydroxy-4-ethoxyphenyl) propenone (3c)}

It was obtained as bright light yellow solid in $85 \%$ yield; m.p.: 140-142 ${ }^{\circ} \mathrm{C}, \mathrm{R}_{\mathrm{f}}: 0.43$ (ethyl acetate: hexane, 3:7). IR $\left(\mathrm{cm}^{-1}\right): 3,708$ (O-H stretch), 2,951 (aliphatic $\mathrm{C}-\mathrm{H}), 1,642(\mathrm{C}=\mathrm{O}), 1,563(\mathrm{C}=\mathrm{C}$ stretch), 1,455 (C-H bending of $\mathrm{CH}_{2}$ ), and 1,258 (C-O). ${ }^{1} \mathrm{HNMR}$ $\left(400 \mathrm{MHz}, \mathrm{CDCl}_{3}\right): \delta 1.41\left(3 \mathrm{H}, \mathrm{t}, \mathrm{J}=6.8, \mathrm{H}-2^{\prime}\right), 4.07\left(2 \mathrm{H}, \mathrm{q}, \mathrm{H}-1^{\prime}\right)$, $2.95\left(3 \mathrm{H}, \mathrm{s}, \mathrm{N}-\mathrm{CH}_{3}\right), 3.12\left(3 \mathrm{H}, \mathrm{s}, \mathrm{N}-\mathrm{CH}_{3}\right), 3.98(2 \mathrm{H}, \mathrm{t}, \mathrm{J}=6.8, \mathrm{H}-$ $\left.1^{\prime}\right), 5.69(1 \mathrm{H}, \mathrm{d}, \mathrm{J}=9.6, \mathrm{H}-2), 6.35-6.39\left(2 \mathrm{H}, \mathrm{m}, \mathrm{H}-5^{\prime \prime}\right.$ and $\left.\mathrm{H}-3^{\prime \prime}\right)$, $7.60\left(1 \mathrm{H}, \mathrm{d}, \mathrm{J}=8.8 \mathrm{H}-6^{\prime \prime}\right)$, and $7.85(1 \mathrm{H}, \mathrm{d}, \mathrm{J}=9.6, \mathrm{H}-3) .{ }^{13} \mathrm{C} \mathrm{NMR}$ $\left(100.6 \mathrm{MHz}_{\mathrm{CDCl}}\right)$ : $190.83(\mathrm{C}-1), 165.56\left(\mathrm{C}-4^{\prime \prime}\right), 164.09\left(\mathrm{C}-2^{\prime \prime}\right)$, 154.07 (C-3), 129.80 (C-6"), 113.77 (C-1" $), 106.86\left(\mathrm{C}-5^{\prime \prime}\right), 101.57$ $\left(\mathrm{C}-3^{\prime \prime}\right), 89.78\left({ }^{2} \mathrm{C}\right), 68.20\left(\mathrm{C}-1^{\prime}\right)$, and $15.07\left(\mathrm{C}-2^{\prime}\right)$. HR-MS for $\mathrm{C}_{13} \mathrm{H}_{17} \mathrm{NO}_{3}[\mathrm{M}+\mathrm{H}]^{+} m / z$ : Calcd 236.1281; Observed 236.1268. The major mass fragments observed were $\mathrm{C}_{9} \mathrm{H}_{9} \mathrm{O}_{3}{ }^{+}(165)$, $\mathrm{C}_{7} \mathrm{H}_{5} \mathrm{O}_{3}{ }^{+}(137), \mathrm{C}_{5} \mathrm{H}_{8} \mathrm{NO}^{+}(98)$, and $\mathrm{C}_{4} \mathrm{H}_{10} \mathrm{~N}^{+}(72)$.

\section{3-Dimethylamino-1-(2-hydroxy- 4-propoxyphenyl) propenone (3d)}

It was obtained as orange yellow solid in $81 \%$ yield; m.p.: 121-124 ${ }^{\circ} \mathrm{C}, \mathrm{R}_{\mathrm{f}}: 0.45$ (ethyl acetate: hexane, 3:7). IR $\left(\mathrm{cm}^{-1}\right)$ : 3,709 (O-H stretch), 2,949 (aliphatic C-H), 1,641 (C=O), 1,569 $\left(\mathrm{C}=\mathrm{C}\right.$ stretch), 1,461 (C-H bending of $\left.\mathrm{CH}_{2}\right)$, and 1,268 (C-O). ${ }^{1} \mathrm{HNMR}\left(400 \mathrm{MHz}, \mathrm{CDCl}_{3}\right): \delta 1.06\left(3 \mathrm{H}, \mathrm{t}, \mathrm{J}=7.2, \mathrm{H}-3^{\prime}\right)$, 1.73-1.88 (2H, m, H-2 $), 2.95\left(3 \mathrm{H}, \mathrm{s}, \mathrm{N}-\mathrm{CH}_{3}\right), 3.12(3 \mathrm{H}, \mathrm{s}, \mathrm{N}-$ $\left.\mathrm{CH}_{3}\right), 3.99\left(2 \mathrm{H}, \mathrm{t}, \mathrm{J}=6.8, \mathrm{H}-1^{\prime}\right), 5.69(1 \mathrm{H}, \mathrm{d}, \mathrm{J}=9.6, \mathrm{H}-2)$, 6.35-6.39 (2H, m, H-5" and H- $\left.3^{\prime \prime}\right), 7.62\left(1 \mathrm{H}, \mathrm{d}, \mathrm{J}=8.8 \mathrm{H}-6^{\prime \prime}\right)$, and $7.84(1 \mathrm{H}, \mathrm{d}, \mathrm{J}=9.6, \mathrm{H}-3) .{ }^{13} \mathrm{C}$ NMR $\left(100.6 \mathrm{MHz}, \mathrm{CDCl}_{3}\right): 190.81$ (C-1), $165.53\left(\mathrm{C}-4^{\prime \prime}\right), 164.06\left(\mathrm{C}-2^{\prime \prime}\right), 154.05$ (C-3), 129.81 (C-6"), $113.62\left(\mathrm{C}-1^{\prime \prime}\right), 106.65\left(\mathrm{C}-5^{\prime \prime}\right), 101.53\left(\mathrm{C}-3^{\prime \prime}\right), 89.75(\mathrm{C}-2), 68.18$ $\left(\mathrm{C}-1^{\prime}\right), 31.07\left(\mathrm{C}-2^{\prime}\right)$, and $15.60\left(\mathrm{C}-3^{\prime}\right)$. HR-MS for $\mathrm{C}_{14} \mathrm{H}_{19} \mathrm{NO}_{3}[\mathrm{M}$ $+\mathrm{H}]^{+} m / z$ : Calcd 250.1437; Observed 250.1424. The major mass fragments observed were $\mathrm{C}_{10} \mathrm{H}_{11} \mathrm{O}_{3}{ }^{+}(179), \mathrm{C}_{7} \mathrm{H}_{5} \mathrm{O}_{3}{ }^{+}(137)$, $\mathrm{C}_{5} \mathrm{H}_{8} \mathrm{NO}^{+}(98)$, and $\mathrm{C}_{4} \mathrm{H}_{10} \mathrm{~N}^{+}(72)$.

\section{3-Dimethylamino-1-(2-hydroxy-4-isopropoxyphenyl) propenone (3e)}

It was obtained as orange yellow solid in $77 \%$ yield; m.p.: 143-145 ${ }^{\circ} \mathrm{C}, \mathrm{R}_{\mathrm{f}}$ : 0.48 (ethyl acetate: hexane, 3:7). IR $\left(\mathrm{cm}^{-1}\right)$ : 3,740 (O-H stretch), 2,954 (aliphatic $\mathrm{C}-\mathrm{H}), 1,647(\mathrm{C}=\mathrm{O}), 1,563$ $\left(\mathrm{C}=\mathrm{C}\right.$ stretch), 1,458 $\left(\mathrm{C}-\mathrm{H}\right.$ bending of $\left.\mathrm{CH}_{2}\right)$, and 1,263 $(\mathrm{C}-\mathrm{O})$. ${ }^{1} \mathrm{HNMR}\left(400 \mathrm{MHz}, \mathrm{CDCl}_{3}\right): \delta 1.30\left(1 \mathrm{H}, \mathrm{d}, \mathrm{J}=4.8 \mathrm{~Hz}, \mathrm{CH}_{3}\right)$, 4.63-4.65 (1H, m, H-1 $\left.{ }^{\prime}\right), 2.95\left(3 \mathrm{H}, \mathrm{s}, \mathrm{N}-\mathrm{CH}_{3}\right), 3.12(3 \mathrm{H}, \mathrm{s}, \mathrm{N}-$ $\left.\mathrm{CH}_{3}\right), 5.69(1 \mathrm{H}, \mathrm{d}, \mathrm{J}=12.4, \mathrm{H}-2), 6.31-6.37\left(2 \mathrm{H}, \mathrm{m}, \mathrm{H}-5^{\prime \prime}\right.$ and $\mathrm{H}-$ $\left.3^{\prime \prime}\right), 7.61\left(1 \mathrm{H}, \mathrm{d}, J=8.8 \mathrm{H}-6^{\prime \prime}\right)$, and $7.82(1 \mathrm{H}, \mathrm{d}, \mathrm{J}=12.4, \mathrm{H}-3) .{ }^{13} \mathrm{C}$ NMR (100.6 MHz, $\left.\mathrm{CDCl}_{3}\right)$ : 190.90 (C-1), $164.93\left(\mathrm{C}-4^{\prime \prime}\right), 164.08$ $\left(\mathrm{C}-2^{\prime \prime}\right), 154.34(\mathrm{C}-3), 130.09\left(\mathrm{C}-6^{\prime \prime}\right), 114.31\left(\mathrm{C}-1^{\prime \prime}\right), 106.91$ (C$\left.5^{\prime \prime}\right), 101.75\left(\mathrm{C}-3^{\prime \prime}\right), 89.87(\mathrm{C}-2), 69.22\left(\mathrm{C}-1^{\prime}\right)$, and $22.65\left(\mathrm{iCH}_{3}\right)$. HR-MS for $\mathrm{C}_{14} \mathrm{H}_{19} \mathrm{NO}_{3}[\mathrm{M}+\mathrm{H}]^{+} m / z$ : Calcd 250.1437; Observed 250.1424. The major mass fragments observed were $\mathrm{C}_{10} \mathrm{H}_{11} \mathrm{O}_{3}{ }^{+}(179), \quad \mathrm{C}_{7} \mathrm{H}_{5} \mathrm{O}_{3}{ }^{+}(137), \mathrm{C}_{5} \mathrm{H}_{8} \mathrm{NO}^{+}(98)$, and $\mathrm{C}_{4} \mathrm{H}_{10} \mathrm{~N}^{+}(72)$.

\section{3-Dimethylamino-1-(4-Butoxy-2-hydroxyphenyl) propenone (3f)}

It was obtained as lemon yellow solid in $88 \%$ yield; m.p.: $103-105^{\circ} \mathrm{C} \mathrm{R}_{\mathrm{f}}: 0.51$ (ethyl acetate: hexane, $\left.3: 7\right)$. IR $\left(\mathrm{cm}^{-1}\right)$ : 3,716 (O-H stretch), 2,955 (aliphatic $\mathrm{C}-\mathrm{H}), 1,638(\mathrm{C}=\mathrm{O}), 1,566(\mathrm{C}=\mathrm{C}$ stretch), 1,465 (C-H bending of $\left.\mathrm{CH}_{2}\right)$, and 1,256 (C-O). ${ }^{1} \mathrm{HNMR}$ $\left(400 \mathrm{MHz}, \mathrm{CDCl}_{3}\right): \delta 0.96\left(3 \mathrm{H}, \mathrm{t}, \mathrm{J}=7.2, \mathrm{H}-4^{\prime}\right), 1.43-1.78(2 \mathrm{H}, \mathrm{m}$, $\mathrm{H}-2^{\prime}$ and $\left.\mathrm{H}-3^{\prime}\right), 2.95\left(3 \mathrm{H}, \mathrm{s}, \mathrm{N}-\mathrm{CH}_{3}\right), 3.16\left(3 \mathrm{H}, \mathrm{s}, \mathrm{N}-\mathrm{CH}_{3}\right), 3.98$ $\left(2 \mathrm{H}, \mathrm{t}, \mathrm{J}=6.8, \mathrm{H}_{-1}^{\prime}\right), 5.63(1 \mathrm{H}, \mathrm{d}, \mathrm{J}=12, \mathrm{H}-2), 6.32-6.38(2 \mathrm{H}, \mathrm{m}$, $\mathrm{H}-5^{\prime \prime}$ and $\left.\mathrm{H}-3^{\prime \prime}\right), 7.55\left(1 \mathrm{H}, \mathrm{d}, \mathrm{J}=8.8 \mathrm{H}-6^{\prime \prime}\right)$, and $7.83(1 \mathrm{H}, \mathrm{d}, \mathrm{J}=$ 12.4, H-3). ${ }^{13} \mathrm{C}$ NMR (100.6 MHz, $\left.\mathrm{CDCl}_{3}\right): 190.79$ (C-1), 165.49 $\left(\mathrm{C}-4^{\prime \prime}\right), 164.05\left(\mathrm{C}-2^{\prime \prime}\right), 154.01(\mathrm{C}-3), 129.76\left(\mathrm{C}-6^{\prime \prime}\right), 113.77\left(\mathrm{C}-1^{\prime \prime}\right)$, $106.80\left(\mathrm{C}-5^{\prime \prime}\right), 101.54\left(\mathrm{C}-3^{\prime \prime}\right), 89.85(\mathrm{C}-2), 68.13\left(\mathrm{C}-1^{\prime}\right), 31.52(\mathrm{C}-$ $\left.2^{\prime}\right), 25.70\left(\mathrm{C}-3^{\prime}\right)$, and $14.29\left(\mathrm{C}-4^{\prime}\right)$. HR-MS for $\mathrm{C}_{15} \mathrm{H}_{21} \mathrm{NO}_{3}[\mathrm{M}+$ $\mathrm{H}]^{+} \mathrm{m} / z$ : Calcd 264.1594; Observed 264.1585. The major mass fragments observed were $\mathrm{C}_{11} \mathrm{H}_{13} \mathrm{O}_{3}{ }^{+}(193), \mathrm{C}_{7} \mathrm{H}_{5} \mathrm{O}_{3}{ }^{+}(137)$, $\mathrm{C}_{5} \mathrm{H}_{8} \mathrm{NO}^{+}(98)$, and $\mathrm{C}_{4} \mathrm{H}_{10} \mathrm{~N}^{+}(72)$.

\section{3-Dimethylamino-1-(4-Pentyloxy-2-hydroxyphenyl) propenone $(3 \mathrm{~g})$}

It was obtained as lemon yellow solid in $78 \%$ yield; m.p.: 98-100 ${ }^{\circ} \mathrm{C}, \mathrm{R}_{\mathrm{f}}: 0.53$ (ethyl acetate: hexane, 3:7). IR ( $\left.\mathrm{cm}^{-1}\right): 3,707$ (O-H stretch), 2,943 (aliphatic $\mathrm{C}-\mathrm{H}), 1,642(\mathrm{C}=\mathrm{O}), 1,576(\mathrm{C}=\mathrm{C}$ stretch), 1,468 (C-H bending of $\mathrm{CH}_{2}$ ), and 1,267 (C-O). ${ }^{1} \mathrm{HNMR}$ $\left(400 \mathrm{MHz}, \mathrm{CDCl}_{3}\right): \delta 0.91\left(3 \mathrm{H}, \mathrm{t}, J=6.8, \mathrm{H}-5^{\prime}\right), 1.31-1.79(6 \mathrm{H}, \mathrm{m}$, $\mathrm{H}-2^{\prime}, \mathrm{H}-3^{\prime}$ and $\left.\mathrm{H}-4^{\prime}\right), 2.95\left(3 \mathrm{H}, \mathrm{s}, \mathrm{N}-\mathrm{CH}_{3}\right), 3.15\left(3 \mathrm{H}, \mathrm{s}, \mathrm{N}-\mathrm{CH}_{3}\right)$, $3.96\left(2 \mathrm{H}, \mathrm{t}, \mathrm{J}=6.8, \mathrm{H}-1^{\prime}\right), 5.66(1 \mathrm{H}, \mathrm{d}, \mathrm{J}=12.4, \mathrm{H}-2), 6.34-6.40$ $\left(2 \mathrm{H}, \mathrm{m}, \mathrm{H}-5^{\prime \prime}\right.$ and $\left.\mathrm{H}-3^{\prime \prime}\right), 7.59\left(1 \mathrm{H}, \mathrm{d}, \mathrm{J}=8.8 \mathrm{H}-6^{\prime \prime}\right)$, and $7.84(1 \mathrm{H}$, $\mathrm{d}, \mathrm{J}=12.4, \mathrm{H}-3) .{ }^{13} \mathrm{C} \mathrm{NMR}\left(100.6 \mathrm{MHz}, \mathrm{CDCl}_{3}\right): 190.83$ (C-1), $165.54\left(\mathrm{C}-4^{\prime \prime}\right), 164.01\left(\mathrm{C}-2^{\prime \prime}\right), 154.03(\mathrm{C}-3), 129.71\left(\mathrm{C}-6^{\prime \prime}\right), 113.70$ $\left(\mathrm{C}-1^{\prime \prime}\right), 106.82\left(\mathrm{C}-5^{\prime \prime}\right), 101.51\left(\mathrm{C}-3^{\prime \prime}\right), 89.83(\mathrm{C}-2), 68.17\left(\mathrm{C}-1^{\prime}\right)$, $31.50\left(\mathrm{C}-2^{\prime}\right), 28.93\left(\mathrm{C}-3^{\prime}\right), 25.66\left(\mathrm{C}-4^{\prime}\right)$, and $14.20\left(\mathrm{C}-5^{\prime}\right)$. HR-MS for $\mathrm{C}_{16} \mathrm{H}_{23} \mathrm{NO}_{3}[\mathrm{M}+\mathrm{H}]^{+} m / z$ : Calcd 278.1750; Observed 278.1765. The major mass fragments observed were $\mathrm{C}_{12} \mathrm{H}_{15} \mathrm{O}_{3}^{+}(207), \quad \mathrm{C}_{7} \mathrm{H}_{5} \mathrm{O}_{3}{ }^{+}(137), \quad \mathrm{C}_{5} \mathrm{H}_{8} \mathrm{NO}^{+}(98), \quad$ and $\mathrm{C}_{4} \mathrm{H}_{10} \mathrm{~N}^{+}(72)$.

\section{3-Dimethylamino-1-(4-Hexyloxy-2-hydroxyphenyl) propenone (3h)}

It was obtained as pale yellow solid in $75 \%$ yield; m.p.: $87-92^{\circ} \mathrm{C}$, $\mathrm{R}_{\mathrm{f}}$ : 0.60 (ethyl acetate: hexane, 3:7). IR $\left(\mathrm{cm}^{-1}\right): 3,718(\mathrm{O}-\mathrm{H}$ stretch), 2,937 (aliphatic C-H), 1,639 (C=O), 1,569 (C=C stretch), 1,470 (C-H bending of $\mathrm{CH}_{2}$ ), and 1,264 (C-O). ${ }^{1} \mathrm{HNMR}\left(400 \mathrm{MHz}, \mathrm{CDCl}_{3}\right): \delta 0.90\left(3 \mathrm{H}, \mathrm{t}, \mathrm{J}=6.8, \mathrm{H}-6^{\prime}\right)$, 1.30-1.79 (8H, m, H-2', H-3', H-4' and H-5'), $2.95(3 \mathrm{H}, \mathrm{s}, \mathrm{N}-$ $\left.\mathrm{CH}_{3}\right), 3.16\left(3 \mathrm{H}, \mathrm{s}, \mathrm{N}-\mathrm{CH}_{3}\right), 3.98\left(2 \mathrm{H}, \mathrm{t}, \mathrm{J}=6.8, \mathrm{H}-1^{\prime}\right), 5.69(1 \mathrm{H}, \mathrm{d}, \mathrm{J}$ $=12, \mathrm{H}-2), 6.35-6.39\left(2 \mathrm{H}, \mathrm{m}, \mathrm{H}-5^{\prime \prime}\right.$ and $\left.\mathrm{H}-3^{\prime \prime}\right), 7.60(1 \mathrm{H}, \mathrm{d}, \mathrm{J}=8.8$ $\left.\mathrm{H}-6^{\prime \prime}\right)$, and $7.84(1 \mathrm{H}, \mathrm{d}, \mathrm{J}=12.4, \mathrm{H}-3) .{ }^{13} \mathrm{C} \mathrm{NMR}(100.6 \mathrm{MHz}$, $\left.\mathrm{CDCl}_{3}\right): 190.80$ (C-1), $165.58\left(\mathrm{C}-4^{\prime \prime}\right), 164.03\left(\mathrm{C}-2^{\prime \prime}\right), 154.05$ (C-3), $129.74\left(\mathrm{C}-6^{\prime \prime}\right), 113.73\left(\mathrm{C}-1^{\prime \prime}\right), 106.86\left(\mathrm{C}-5^{\prime \prime}\right), 101.53\left(\mathrm{C}-3^{\prime \prime}\right), 89.87$ (C-2), $68.19\left(\mathrm{C}-1^{\prime}\right), 31.57\left(\mathrm{C}-2^{\prime}\right), 28.95\left(\mathrm{C}-3^{\prime}\right), 25.67\left(\mathrm{C}-4^{\prime}\right), 22.65$ $\left(\mathrm{C}-5^{\prime}\right)$, and $14.19\left(\mathrm{C}-6^{\prime}\right)$. HR-MS for $\mathrm{C}_{17} \mathrm{H}_{25} \mathrm{NO}_{3}[\mathrm{M}+\mathrm{H}]^{+} m / z$ : Calcd 292.1907; Observed 292.1912. The major mass fragments observed were $\mathrm{C}_{13} \mathrm{H}_{17} \mathrm{O}_{3}{ }^{+}(221), \mathrm{C}_{7} \mathrm{H}_{5} \mathrm{O}_{3}{ }^{+}(137), \mathrm{C}_{5} \mathrm{H}_{8} \mathrm{NO}^{+}(98)$, and $\mathrm{C}_{4} \mathrm{H}_{10} \mathrm{~N}^{+}(72)$. 


\section{3-Dimethylamino-1-(4-Heptyloxy-2-hydroxyphenyl) propenone (3i)}

It was obtained as pale yellow solid in $80 \%$ yield; m.p.: $90-92^{\circ} \mathrm{C}$, $\mathrm{R}_{\mathrm{f}}: 0.50$ (ethyl acetate: hexane, 3:7). IR $\left(\mathrm{cm}^{-1}\right): 3,709(\mathrm{O}-\mathrm{H}$ stretch), 2,941 (aliphatic C-H), 1,631 (C=O), 1,572 (C=C stretch), 1,472 (C-H bending of $\left.\mathrm{CH}_{2}\right)$, and 1,266 (C-O). ${ }^{1} \mathrm{HNMR}\left(400 \mathrm{MHz}, \mathrm{CDCl}_{3}\right): \delta 0.88\left(3 \mathrm{H}, \mathrm{t}, \mathrm{J}=6.8, \mathrm{H}-7^{\prime}\right)$, 1.23-1.78 (10H, m, H-2', H-3', H- $4^{\prime}, \mathrm{H}-5^{\prime}$ and $\left.\mathrm{H}-6^{\prime}\right), 2.92(3 \mathrm{H}$, s, N-CH $\left.\mathrm{CH}_{3}\right), 3.14\left(3 \mathrm{H}, \mathrm{s}, \mathrm{N}-\mathrm{CH}_{3}\right), 3.95\left(2 \mathrm{H}, \mathrm{t}, \mathrm{J}=6.8, \mathrm{H}-\mathrm{1}^{\prime}\right), 5.66$ $(1 \mathrm{H}, \mathrm{d}, \mathrm{J}=12, \mathrm{H}-2), 6.35-6.39\left(2 \mathrm{H}, \mathrm{m}, \mathrm{H}-5^{\prime \prime}\right.$ and $\left.\mathrm{H}-3^{\prime \prime}\right), 7.55(1 \mathrm{H}$, $\left.\mathrm{d}, \mathrm{J}=8.8 \mathrm{H}-6^{\prime \prime}\right)$, and $7.82(1 \mathrm{H}, \mathrm{d}, \mathrm{J}=12.4, \mathrm{H}-3) .{ }^{13} \mathrm{C} \mathrm{NMR}$ $\left(100.6 \mathrm{MHz}, \mathrm{CDCl}_{3}\right): 190.81(\mathrm{C}-1), 165.59\left(\mathrm{C}-4^{\prime \prime}\right), 164.05\left(\mathrm{C}-2^{\prime \prime}\right)$, 154.07 (C-3), $129.72\left(\mathrm{C}-6^{\prime \prime}\right), 113.74\left(\mathrm{C}-1^{\prime \prime}\right), 106.89\left(\mathrm{C}-5^{\prime \prime}\right), 101.54$ $\left(\mathrm{C}-3^{\prime \prime}\right), 89.89(\mathrm{C}-2), 68.16\left(\mathrm{C}-1^{\prime}\right), 31.57-22.66\left(\mathrm{C}-2^{\prime}, \mathrm{C}-3^{\prime}, \mathrm{C}-4^{\prime}\right.$, C-5', C-6 $\left.6^{\prime}\right)$, and $14.20\left(\mathrm{C}-7^{\prime}\right)$. HR-MS for $\mathrm{C}_{18} \mathrm{H}_{27} \mathrm{NO}_{3}[\mathrm{M}+$ $\mathrm{H}]^{+} m / z$ : Calcd 306.2063; Observed 306.2059. The major mass fragments observed were $\mathrm{C}_{14} \mathrm{H}_{19} \mathrm{O}_{3}{ }^{+}(235), \mathrm{C}_{7} \mathrm{H}_{5} \mathrm{O}_{3}{ }^{+}(137)$, $\mathrm{C}_{5} \mathrm{H}_{8} \mathrm{O}^{+}(98)$, and $\mathrm{C}_{4} \mathrm{H}_{10} \mathrm{~N}^{+}(72)$.

\section{3-Dimethylamino-1-(2-hydroxy-4-octyloxyphenyl) propenone (3j)}

It was obtained as yellow solid in $76 \%$ yield; m.p.: $70-72^{\circ} \mathrm{C}, \mathrm{R}_{\mathrm{f}}: 0.45$ (ethyl acetate: hexane, 3:7). IR $\left(\mathrm{cm}^{-1}\right)$ : 3,718 (O-H stretch), 2,936 (aliphatic C-H), 1,641 $(\mathrm{C}=\mathrm{O}), 1,579(\mathrm{C}=\mathrm{C}$ stretch), 1,475 $(\mathrm{C}-\mathrm{H}$ bending of $\mathrm{CH}_{2}$ ), and 1,262 (C-O). ${ }^{1} \mathrm{HNMR}\left(400 \mathrm{MHz}, \mathrm{CDCl}_{3}\right): \delta$ $0.90\left(3 \mathrm{H}, \mathrm{t}, \mathrm{J}=6.8, \mathrm{H}-8^{\prime}\right), 1.24-1.78\left(12 \mathrm{H}, \mathrm{m}, \mathrm{H}-2^{\prime}, \mathrm{H}-3^{\prime}, \mathrm{H}-4^{\prime}, \mathrm{H}-\right.$ $5^{\prime}, \mathrm{H}-6^{\prime}$, and $\left.\mathrm{H}-7^{\prime}\right), 2.92\left(3 \mathrm{H}, \mathrm{s}, \mathrm{N}-\mathrm{CH}_{3}\right), 3.13\left(3 \mathrm{H}, \mathrm{s}, \mathrm{N}-\mathrm{CH}_{3}\right), 3.94$ $\left(2 \mathrm{H}, \mathrm{t}, \mathrm{J}=6.8, \mathrm{H}_{-1}^{\prime}\right), 5.67(1 \mathrm{H}, \mathrm{d}, \mathrm{J}=12, \mathrm{H}-3), 6.35-6.39(2 \mathrm{H}, \mathrm{m}, \mathrm{H}-$ $5^{\prime \prime}$ and $\left.\mathrm{H}-3^{\prime \prime}\right), 7.58\left(1 \mathrm{H}, \mathrm{d}, \mathrm{J}=8.8 \mathrm{H}-6^{\prime \prime}\right)$, and $7.82(1 \mathrm{H}, \mathrm{d}, \mathrm{J}=12.4$, $\mathrm{H}-2) .{ }^{13} \mathrm{C} \mathrm{NMR}\left(100.6 \mathrm{MHz}, \mathrm{CDCl}_{3}\right): 190.80$ (C-1), 165.57 (C-4"), 164.07 (C-2"), $154.04(\mathrm{C}-3), 129.74\left(\mathrm{C}-6^{\prime \prime}\right), 113.72\left(\mathrm{C}-1^{\prime \prime}\right), 106.90$ $\left(\mathrm{C}-5^{\prime \prime}\right), 101.56\left(\mathrm{C}-3^{\prime \prime}\right), 89.90(\mathrm{C}-2), 68.15\left(\mathrm{C}-1^{\prime}\right), 31.86-22.65$ (C$\left.2^{\prime}, \mathrm{C}-3^{\prime}, \mathrm{C}-4^{\prime}, \mathrm{C}-5^{\prime}, \mathrm{C}-6^{\prime}, \mathrm{C}-7^{\prime}\right)$, and $14.46\left(\mathrm{C}-8^{\prime}\right)$. HR-MS for $\mathrm{C}_{19} \mathrm{H}_{29} \mathrm{NO}_{3}[\mathrm{M}+\mathrm{H}]^{+} m / z$ : Calcd 320.2220; Observed 320.2214. The major mass fragments observed were $\mathrm{C}_{15} \mathrm{H}_{21} \mathrm{O}_{3}{ }^{+}(249)$, $\mathrm{C}_{7} \mathrm{H}_{5} \mathrm{O}_{3}{ }^{+}(137), \mathrm{C}_{5} \mathrm{H}_{8} \mathrm{NO}^{+}(98)$, and $\mathrm{C}_{4} \mathrm{H}_{10} \mathrm{~N}^{+}(72)$.

\section{3-Dimethylamino-1-(2-hydroxy-4-nonyloxyphenyl) propenone (3k)}

It was obtained as yellow solid in $86 \%$ yield; m.p.: $78-80^{\circ} \mathrm{C}, \mathrm{R}_{\mathrm{f}}$ : 0.49 (ethyl acetate: hexane, 3:7). IR $\left(\mathrm{cm}^{-1}\right): 3,711(\mathrm{O}-\mathrm{H}$ stretch), 2,945 (aliphatic $\mathrm{C}-\mathrm{H}), 1,642(\mathrm{C}=\mathrm{O}), 1,593(\mathrm{C}=\mathrm{C}$ stretch), 1,478 (C-H bending of $\mathrm{CH}_{2}$ ), and 1,265 (C-O). ${ }^{1} \mathrm{HNMR}(400 \mathrm{MHz}$, $\left.\mathrm{CDCl}_{3}\right): \delta 0.86\left(3 \mathrm{H}, \mathrm{t}, \mathrm{J}=6.8, \mathrm{H}-9^{\prime}\right), 1.26-1.78\left(12 \mathrm{H}, \mathrm{m}, \mathrm{H}-2^{\prime}, \mathrm{H}-\right.$ $3^{\prime}, \mathrm{H}-4^{\prime}, \mathrm{H}-5^{\prime}, \mathrm{H}-6^{\prime}, \mathrm{H}-7^{\prime}$, and $\left.\mathrm{H}-8^{\prime}\right), 2.92\left(3 \mathrm{H}, \mathrm{s}, \mathrm{N}-\mathrm{CH}_{3}\right), 3.13$ $\left(3 \mathrm{H}, \mathrm{s}, \mathrm{N}-\mathrm{CH}_{3}\right), 3.98\left(2 \mathrm{H}, \mathrm{t}, \mathrm{J}=6.8, \mathrm{H}-1^{\prime}\right), 5.64(1 \mathrm{H}, \mathrm{d}, \mathrm{J}=12, \mathrm{H}-$ 2), 6.35-6.39 (2H, m, H-5" and H-3" $), 7.59\left(1 \mathrm{H}, \mathrm{d}, \mathrm{J}=8.8 \mathrm{H}-6^{\prime \prime}\right)$, and $7.87(1 \mathrm{H}, \mathrm{d}, \mathrm{J}=12.4, \mathrm{H}-3) .{ }^{13} \mathrm{C} \mathrm{NMR}\left(100.6 \mathrm{MHz}, \mathrm{CDCl}_{3}\right)$ : 190.77 (C-1), $165.56\left(\mathrm{C}-4^{\prime \prime}\right), 164.03$ (C-2"), 154.01 (C-3), 129.75 $\left(\mathrm{C}-6^{\prime \prime}\right), 113.69\left(\mathrm{C}-1^{\prime \prime}\right), 106.91\left(\mathrm{C}-5^{\prime \prime}\right), 101.55\left(\mathrm{C}-3^{\prime \prime}\right), 89.86(\mathrm{C}-2)$, $68.14\left(\mathrm{C}-1^{\prime}\right), 31.88-22.63\left(\mathrm{C}-2^{\prime}, \mathrm{C}-3^{\prime}, \mathrm{C}-4^{\prime}, \mathrm{C}-5^{\prime}, \mathrm{C}-6^{\prime}, \mathrm{C}-7^{\prime}, \mathrm{C}-\right.$ $8^{\prime}$ ), and $14.45\left(\mathrm{C}-9^{\prime}\right)$. HR-MS for $\mathrm{C}_{20} \mathrm{H}_{31} \mathrm{NO}_{3}[\mathrm{M}+\mathrm{H}]^{+} m / z$ : Calcd 334.2376; Observed 334.2382. The major mass fragments observed were $\mathrm{C}_{16} \mathrm{H}_{23} \mathrm{O}_{3}{ }^{+}(263), \mathrm{C}_{7} \mathrm{H}_{5} \mathrm{O}_{3}{ }^{+}(137), \mathrm{C}_{5} \mathrm{H}_{8} \mathrm{NO}^{+}(98)$, and $\mathrm{C}_{4} \mathrm{H}_{10} \mathrm{~N}^{+}(72)$.

\section{3-Dimethylamino 1-(4-decyloxy-2-hydroxyphenyl) propenone (3l)}

It was obtained as yellow solid in $83 \%$ yield; m.p.:76-79 ${ }^{\circ} \mathrm{C}, \mathrm{R}_{\mathrm{f}}: 0.54$ (ethyl acetate: hexane, 3:7). IR $\left(\mathrm{cm}^{-1}\right): 3,708$ (O-H stretch), 2,955 (aliphatic C-H), 1,629 (C=O), 1,586 (C=C stretch), 1,498 (C-H bending of $\left.\mathrm{CH}_{2}\right)$, and $1,272(\mathrm{C}-\mathrm{O}) .{ }^{1} \mathrm{HNMR}\left(400 \mathrm{MHz}, \mathrm{CDCl}_{3}\right): \delta$ $0.86\left(3 \mathrm{H}, \mathrm{t}, \mathrm{J}=6.8, \mathrm{H}-10^{\prime}\right), 1.25-1.78\left(16 \mathrm{H}, \mathrm{m}, \mathrm{H}-2^{\prime}, \mathrm{H}-3^{\prime}, \mathrm{H}-4^{\prime}\right.$, $\mathrm{H}-5^{\prime}, \mathrm{H}-6^{\prime}, \mathrm{H}-7^{\prime}, \mathrm{H}-8^{\prime}$, and $\left.\mathrm{H}-9^{\prime}\right), 2.92\left(3 \mathrm{H}, \mathrm{s}, \mathrm{N}-\mathrm{CH}_{3}\right), 3.13(3 \mathrm{H}$, $\left.\mathrm{s}, \mathrm{N}-\mathrm{CH}_{3}\right), 3.94\left(2 \mathrm{H}, \mathrm{t}, \mathrm{J}=6.8 \mathrm{H}^{-1}{ }^{\prime}\right) 5.68(1 \mathrm{H}, \mathrm{d}, \mathrm{J}=12, \mathrm{H}-2)$, 6.33-6.37 (2H, m, H-5 ${ }^{\prime \prime}$ and $\left.\mathrm{H}-3^{\prime \prime}\right) 7.56\left(1 \mathrm{H}, \mathrm{d}, \mathrm{J}=8.8 \mathrm{H}-6^{\prime \prime}\right)$, and $7.83(1 \mathrm{H}, \mathrm{d}, \mathrm{J}=12.4, \mathrm{H}-3) .{ }^{13} \mathrm{C} \mathrm{NMR}\left(100.6 \mathrm{MHz}, \mathrm{CDCl}_{3}\right): 190.81$ (C-1), $165.59\left(\mathrm{C}-4^{\prime \prime}\right), 164.05\left(\mathrm{C}-2^{\prime \prime}\right), 154.03(\mathrm{C}-3), 129.73\left(\mathrm{C}-6^{\prime \prime}\right)$, $113.74\left(\mathrm{C}-1^{\prime \prime}\right), 106.93\left(\mathrm{C}-5^{\prime \prime}\right), 101.58\left(\mathrm{C}-3^{\prime \prime}\right), 89.89$ (C-2), 68.18 $\left(\mathrm{C}-1^{\prime}\right), 31.97-22.76\left(\mathrm{C}-3^{\prime}, \mathrm{C} 4^{\prime}, \mathrm{C} 5^{\prime}, \mathrm{C}-6^{\prime}, \mathrm{C}-7^{\prime}, \mathrm{C}-8^{\prime}, \mathrm{C}-9^{\prime}\right)$, and $14.19\left(\mathrm{C}-10^{\prime}\right)$. HR-MS for $\mathrm{C}_{21} \mathrm{H}_{33} \mathrm{NO}_{3}[\mathrm{M}+\mathrm{H}]^{+} m / z$ : Calcd 348.2533; Observed 348.2550. The major mass fragments observed were $\mathrm{C}_{17} \mathrm{H}_{25} \mathrm{O}_{3}{ }^{+}(277), \mathrm{C}_{7} \mathrm{H}_{5} \mathrm{O}_{3}{ }^{+}(137), \mathrm{C}_{5} \mathrm{H}_{8} \mathrm{NO}^{+}$(98), and $\mathrm{C}_{4} \mathrm{H}_{10} \mathrm{~N}^{+}(72)$.

\section{3-Dimethylamino 1-(4-dodecyloxy-2-hydroxyphenyl) propenone $(3 \mathrm{~m})$}

It was obtained as yellow solid in $81 \%$ yield; m.p.: $86-88^{\circ} \mathrm{C}, \mathrm{R}_{\mathrm{f}}$ : 0.51 (ethyl acetate: hexane, $3: 7)$. IR $\left(\mathrm{cm}^{-1}\right): 3,757$ (O-H stretch), 2,945 (aliphatic C-H), 1,634 (C=O), 1,539 (C=C stretch), 1,495 (C-H bending of $\left.\mathrm{CH}_{2}\right)$, and $1,278(\mathrm{C}-\mathrm{O}) .{ }^{1} \mathrm{HNMR}(400 \mathrm{MHz}$, $\left.\mathrm{CDCl}_{3}\right): \delta 0.87\left(3 \mathrm{H}, \mathrm{t}, \mathrm{J}=6.8, \mathrm{H}-12^{\prime}\right), 1.26-1.77\left(20 \mathrm{H}, \mathrm{m}, \mathrm{H}-2^{\prime}, \mathrm{H}-\right.$ $3^{\prime}, \mathrm{H}-4^{\prime}, \mathrm{H}-5^{\prime}, \mathrm{H}-6^{\prime}, \mathrm{H}-7^{\prime}, \mathrm{H}-8^{\prime}, \mathrm{H}-9^{\prime}, \mathrm{H}-10^{\prime}$, and $\left.\mathrm{H}-11^{\prime}\right), 2.93$ $\left(3 \mathrm{H}, \mathrm{s}, \mathrm{N}-\mathrm{CH}_{3}\right), 3.15\left(3 \mathrm{H}, \mathrm{s}, \mathrm{N}-\mathrm{CH}_{3}\right), 3.94\left(2 \mathrm{H}, \mathrm{t}, \mathrm{J}=6.8, \mathrm{H}-\mathrm{1}^{\prime}\right)$, $5.69(1 \mathrm{H}, \mathrm{d}, \mathrm{J}=12, \mathrm{H}-2), 6.33-6.37\left(2 \mathrm{H}, \mathrm{m}, \mathrm{H}-5^{\prime \prime}\right.$ and $\left.\mathrm{H}-3^{\prime \prime}\right) 7.58$ $\left(1 \mathrm{H}, \mathrm{d}, \mathrm{J}=7.2, \mathrm{H}-6^{\prime \prime}\right)$, and $7.84(1 \mathrm{H}, \mathrm{d}, \mathrm{J}=12.4, \mathrm{H}-3) .{ }^{13} \mathrm{C} \mathrm{NMR}$ (100.6 MHz, $\left.\mathrm{CDCl}_{3}\right)$ : 190.67 (C-1), $165.59\left(\mathrm{C}-4^{\prime \prime}\right), 164.04\left(\mathrm{C}-2^{\prime \prime}\right)$, 154.03 (C-3), $129.74\left(\mathrm{C}-6^{\prime \prime}\right), 113.73\left(\mathrm{C}-1^{\prime \prime}\right), 106.93\left(\mathrm{C}-5^{\prime \prime}\right), 101.57$ $\left(\mathrm{C}-3^{\prime \prime}\right), 89.89$ (C-2), 68.18 (C-1'), 31.94-22.73 (C-3', C-4', C-5', C-6 $\left.{ }^{\prime}, \mathrm{C}-7^{\prime}, \mathrm{C}-8^{\prime}, \mathrm{C}-9^{\prime}, \mathrm{C}-10^{\prime}, \mathrm{C}-11^{\prime}\right)$, and 14.20 (C-12'). HR-MS for $\mathrm{C}_{23} \mathrm{H}_{37} \mathrm{NO}_{3}[\mathrm{M}+\mathrm{H}]^{+} m / z$ : Calcd 376.2846; Observed 376.2858. The major mass fragments observed were $\mathrm{C}_{19} \mathrm{H}_{29} \mathrm{O}_{3}{ }^{+}(305), \mathrm{C}_{7} \mathrm{H}_{5} \mathrm{O}_{3}{ }^{+}(137), \mathrm{C}_{5} \mathrm{H}_{8} \mathrm{O}^{+}(98)$, and $\mathrm{C}_{4} \mathrm{H}_{10} \mathrm{~N}^{+}(72)$.

\section{3-Dimethylamino 1-(4-tridecyloxy-2-hydroxyphenyl) propenone (3n)}

It was obtained as yellow solid in $72 \%$ yield; m.p.: $87-90^{\circ} \mathrm{C}, \mathrm{R}_{\mathrm{f}}$ : 0.49 (ethyl acetate: hexane, $3: 7)$. IR $\left(\mathrm{cm}^{-1}\right): 3,770$ (O-H stretch), 2,941 (aliphatic C-H), 1,630 (C=O), 1,545 (C=C stretch), 1,497 (C-H bending of $\mathrm{CH}_{2}$ ), and $1,287(\mathrm{C}-\mathrm{O}) .{ }^{1} \mathrm{HNMR}(400 \mathrm{MHz}$, $\left.\mathrm{CDCl}_{3}\right): \delta 0.86\left(3 \mathrm{H}, \mathrm{t}, \mathrm{J}=6.8, \mathrm{H}-13^{\prime}\right), 1.24-1.79\left(22 \mathrm{H}, \mathrm{m}, \mathrm{H}-2^{\prime}\right.$, $\mathrm{H}-3^{\prime}, \mathrm{H}-4^{\prime}, \mathrm{H}-5^{\prime}, \mathrm{H}-6^{\prime}, \mathrm{H}-7^{\prime}, \mathrm{H}-8^{\prime}, \mathrm{H}-9^{\prime}, \mathrm{H}-10^{\prime}, \mathrm{H}-11^{\prime}$, and $\mathrm{H}-$ $\left.12^{\prime}\right), 2.92\left(3 \mathrm{H}, \mathrm{s}, \mathrm{N}-\mathrm{CH}_{3}\right), 3.14\left(3 \mathrm{H}, \mathrm{s}, \mathrm{N}-\mathrm{CH}_{3}\right), 3.98(2 \mathrm{H}, \mathrm{t}, \mathrm{J}=$ $\left.6.8, \mathrm{H}^{\prime} \mathrm{1}^{\prime}\right), 5.66(1 \mathrm{H}, \mathrm{d}, \mathrm{J}=12, \mathrm{H}-2), 6.32-6.35\left(2 \mathrm{H}, \mathrm{m}, \mathrm{H}-5^{\prime \prime}\right.$ and $\left.\mathrm{H}-3^{\prime \prime}\right), 7.56\left(1 \mathrm{H}, \mathrm{d}, \mathrm{J}=8.8, \mathrm{H}-6^{\prime \prime}\right)$, and $7.80(1 \mathrm{H}, \mathrm{d}, \mathrm{J}=12.4, \mathrm{H}-3)$. ${ }^{13} \mathrm{C}$ NMR (100.6 MHz, $\left.\mathrm{CDCl}_{3}\right): 190.69(\mathrm{C}-1), 165.60\left(\mathrm{C}-4^{\prime \prime}\right)$, 164.05 (C-2"), 154.02 (C-3), $129.73\left(\mathrm{C}-6^{\prime \prime}\right), 113.74\left(\mathrm{C}-1^{\prime \prime}\right)$, $106.94\left(\mathrm{C}-5^{\prime \prime}\right), 101.58\left(\mathrm{C}-3^{\prime \prime}\right), 89.90(\mathrm{C}-2), 68.18\left(\mathrm{C}-1^{\prime}\right)$, 32-22.77 (C-3', C4', C5', C-6 ${ }^{\prime}, \mathrm{C}-7^{\prime}, \mathrm{C}-8^{\prime}, \mathrm{C}-9^{\prime}, \mathrm{C}-10^{\prime}, \mathrm{C}-11^{\prime}$, C-12'), and $14.19\left(\mathrm{C}-13^{\prime}\right)$. HR-MS for $\mathrm{C}_{24} \mathrm{H}_{39} \mathrm{NO}_{3}[\mathrm{M}+\mathrm{H}]^{+} m / z$ : Calcd 390.3002; Observed 390.3010. The major mass fragments 
observed were $\mathrm{C}_{20} \mathrm{H}_{31} \mathrm{O}_{3}{ }^{+}(319), \mathrm{C}_{7} \mathrm{H}_{5} \mathrm{O}_{3}{ }^{+}(137), \mathrm{C}_{5} \mathrm{H}_{8} \mathrm{NO}^{+}(98)$, and $\mathrm{C}_{4} \mathrm{H}_{10} \mathrm{~N}^{+}(72)$.

\section{3-Dimethylamino}

1-(4-tetradecyloxy-2-hydroxyphenyl)propenone (30)

It was obtained as yellow solid in $78 \%$ yield; m.p.: $76-81^{\circ} \mathrm{C}, \mathrm{R}_{\mathrm{f}}$ : 0.50 (ethyl acetate: hexane, $3: 7)$. IR $\left(\mathrm{cm}^{-1}\right): 3,763$ (O-H stretch), 2,934 (aliphatic $\mathrm{C}-\mathrm{H}), 1,645(\mathrm{C}=\mathrm{O}), 1,536(\mathrm{C}=\mathrm{C}$ stretch), 1,489 (C-H bending of $\mathrm{CH}_{2}$ ), and 1,278 (C-O). ${ }^{1} \mathrm{HNMR}(400 \mathrm{MHz}$, $\left.\mathrm{CDCl}_{3}\right): \delta 0.87\left(3 \mathrm{H}, \mathrm{t}, \mathrm{J}=6.8, \mathrm{H}-14^{\prime}\right), 1.26-1.80\left(24 \mathrm{H}, \mathrm{m}, \mathrm{H}-2^{\prime}\right.$, $\mathrm{H}-3^{\prime}, \mathrm{H}-4^{\prime}, \mathrm{H}-5^{\prime}, \mathrm{H}-6^{\prime}, \mathrm{H}-7^{\prime}, \mathrm{H}-8^{\prime}, \mathrm{H}-9^{\prime}, \mathrm{H}-10^{\prime}, \mathrm{H}-11^{\prime} \mathrm{H}-12^{\prime}$, and $\left.\mathrm{H}-13^{\prime}\right), 2.92\left(3 \mathrm{H}, \mathrm{s}, \mathrm{N}-\mathrm{CH}_{3}\right), 3.19\left(3 \mathrm{H}, \mathrm{s}, \mathrm{N}-\mathrm{CH}_{3}\right), 3.99(2 \mathrm{H}$, $\left.\mathrm{t}, \mathrm{J}=6.8, \mathrm{H}-\mathrm{1}^{\prime}\right), 5.69(1 \mathrm{H}, \mathrm{d}, \mathrm{J}=12, \mathrm{H}-2), 6.31-6.36(2 \mathrm{H}, \mathrm{m}, \mathrm{H}-$ $5^{\prime \prime}$ and $\left.\mathrm{H}-3^{\prime \prime}\right), 7.55\left(1 \mathrm{H}, \mathrm{d}, \mathrm{J}=8.8, \mathrm{H}-6^{\prime \prime}\right)$, and $7.83(1 \mathrm{H}, \mathrm{d}, \mathrm{J}=$ 12.4, H-3). ${ }^{13} \mathrm{C}$ NMR (100.6 MHz, $\mathrm{CDCl}_{3}$ ): 190.71 (C-1), 165.57 (C-4"), 164.03 (C-2"), 154.08 (C-3), $129.71\left(\mathrm{C}-6^{\prime \prime}\right), 113.70(\mathrm{C}-$ $\left.1^{\prime \prime}\right), 106.92\left(\mathrm{C}-5^{\prime \prime}\right), 101.55$ (C-3"), $89.93(\mathrm{C}-2), 68.15\left(\mathrm{C}-1^{\prime}\right)$,

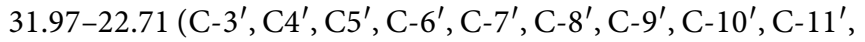
C-12', C-13'), and $14.16\left(\mathrm{C}-14^{\prime}\right)$. HR-MS for $\mathrm{C}_{25} \mathrm{H}_{41} \mathrm{NO}_{3}[\mathrm{M}+$ $\mathrm{H}]^{+} m / z$ : Calcd 404.3159; Observed 404.3143. The major mass fragments observed were $\mathrm{C}_{21} \mathrm{H}_{33} \mathrm{O}_{3}{ }^{+}(333), \mathrm{C}_{7} \mathrm{H}_{5} \mathrm{O}_{3}{ }^{+}(137)$, $\mathrm{C}_{5} \mathrm{H}_{8} \mathrm{NO}^{+}(98)$, and $\mathrm{C}_{4} \mathrm{H}_{10} \mathrm{~N}^{+}(72)$.

\section{3-Dimethylamino 1-(5-bromo-2-hydroxyphenyl) propenone (3p)}

It was obtained as bright yellow solid in $87 \%$ yield; m.p.: $128-132^{\circ} \mathrm{C}, \mathrm{R}_{\mathrm{f}}: 0.50$ (ethyl acetate: hexane, $\left.3: 7\right)$. IR $\left(\mathrm{cm}^{-1}\right)$ : 3,735 (O-H stretch), 2,923 (aliphatic $\mathrm{C}-\mathrm{H}), 1,638(\mathrm{C}=\mathrm{O})$, $1,548\left(\mathrm{C}=\mathrm{C}\right.$ stretch), $1,437\left(\mathrm{C}-\mathrm{H}\right.$ bending of $\left.\mathrm{CH}_{2}\right)$, and 1,258 (C-O). ${ }^{1} \mathrm{HNMR}\left(400 \mathrm{MHz}, \mathrm{CDCl}_{3}\right): \delta 2.95\left(3 \mathrm{H}, \mathrm{s}, \mathrm{N}-\mathrm{CH}_{3}\right), 3.18$ $\left(3 \mathrm{H}, \mathrm{s}, \mathrm{N}-\mathrm{CH}_{3}\right), 5.68(1 \mathrm{H}, \mathrm{d}, \mathrm{J}=9.6, \mathrm{H}-2), 7.06(1 \mathrm{H}, \mathrm{d}, \mathrm{J}=$ $\left.7.2 \mathrm{~Hz}, \mathrm{H}-3^{\prime \prime}\right) 7.43-7.47\left(1 \mathrm{H}, \mathrm{m}, \mathrm{H}-4^{\prime \prime}\right), 7.78(1 \mathrm{H}, \mathrm{d}, \mathrm{J}=2, \mathrm{H}-$ $\left.6^{\prime \prime}\right)$, and $7.90(1 \mathrm{H}, \mathrm{d}, \mathrm{J}=9.6, \mathrm{H}-3) \cdot{ }^{13} \mathrm{C}$ NMR $(100.6 \mathrm{MHz}$, $\mathrm{CDCl}_{3}$ ): 190.75 (C-1), 164.10 (C-2'), 154.06 (C-3), 134.44 (C$\left.4^{\prime}\right), 129.56\left(\mathrm{C}-6^{\prime}\right), 124.63\left(\mathrm{C}-1^{\prime}\right), 123.20\left(\mathrm{C}-3^{\prime}\right), 118.73\left(\mathrm{C}-5^{\prime}\right)$, and $89.93(\mathrm{C}-2)$. HR-MS for $\mathrm{C}_{11} \mathrm{H}_{11} \mathrm{BrNO}_{2}[\mathrm{M}+\mathrm{H}]^{+} m / z$ : Calcd 269.0045; Observed 269.0029. The major mass fragments observed were $\mathrm{C}_{7} \mathrm{H}_{3} \mathrm{BrO}_{2}{ }^{+}(197), \mathrm{C}_{5} \mathrm{H}_{8} \mathrm{NO}^{+}(98)$, and $\mathrm{C}_{4} \mathrm{H}_{10} \mathrm{~N}^{+}(72)$.

\section{3-Dimethylamino-1-(5-chloro-2-hydroxyphenyl) propenone (3q)}

It was obtained as bright yellow solid in $78 \%$ yield; m.p.: $125-128^{\circ} \mathrm{C}, \mathrm{R}_{\mathrm{f}}: 0.53$ (ethyl acetate: hexane, $\left.3: 7\right)$. IR $\left(\mathrm{cm}^{-1}\right)$ : 3,739 (O-H stretch), 2,925 (aliphatic $\mathrm{C}-\mathrm{H}), 1,644(\mathrm{C}=\mathrm{O})$, $1,555\left(\mathrm{C}=\mathrm{C}\right.$ stretch), $1,429\left(\mathrm{C}-\mathrm{H}\right.$ bending of $\left.\mathrm{CH}_{2}\right)$, and 1,258 (C-O). ${ }^{1} \mathrm{HNMR}\left(400 \mathrm{MHz}, \mathrm{CDCl}_{3}\right): \delta 2.93\left(3 \mathrm{H}, \mathrm{s}, \mathrm{N}-\mathrm{CH}_{3}\right), 3.19$ $\left(3 \mathrm{H}, \mathrm{s}, \mathrm{N}-\mathrm{CH}_{3}\right), 5.69(1 \mathrm{H}, \mathrm{d}, \mathrm{J}=9.6, \mathrm{H}-2), 6.81(1 \mathrm{H}, \mathrm{d}, \mathrm{J}=$ $\left.7.2 \mathrm{~Hz}, \mathrm{H}-3^{\prime \prime}\right), 7.01-7.05\left(1 \mathrm{H}, \mathrm{m}, \mathrm{H}-4^{\prime \prime}\right), 7.69(1 \mathrm{H}, \mathrm{d}, \mathrm{J}=2, \mathrm{H}-$ $\left.6^{\prime \prime}\right)$, and $7.84(1 \mathrm{H}, \mathrm{d}, \mathrm{J}=9.6, \mathrm{H}-3) .{ }^{13} \mathrm{C}$ NMR $(100.6 \mathrm{MHz}$, $\mathrm{CDCl}_{3}$ ): 190.63 (C-1), 158.67 (C-2), 154.19 (C-2"), 134.45 (C$\left.4^{\prime \prime}\right), 131.26$ (C-6"), 130.55 (C-5"), $121.10\left(\mathrm{C}-1^{\prime \prime}\right), 119.25\left(\mathrm{C}-3^{\prime \prime}\right)$, and $86.91(\mathrm{C}-3)$. HR-MS for $\mathrm{C}_{12} \mathrm{H}_{13} \mathrm{ClNO}_{2}[\mathrm{M}+\mathrm{H}]^{+} m / z$ : Calcd 239.0707; Observed 239.0712. The major mass fragments observed were $\mathrm{C}_{7} \mathrm{H}_{3} \mathrm{ClO}_{2}{ }^{+}(153), \mathrm{C}_{5} \mathrm{H}_{8} \mathrm{NO}^{+}(98)$, and $\mathrm{C}_{4} \mathrm{H}_{10} \mathrm{~N}^{+}(72)$.

\section{3-Dimethylamino 1-(3,5-dichloro-2-hydroxyphenyl) propenone (3r)}

It was obtained as bright yellow solid in $82 \%$ yield; m.p.: $137-141^{\circ} \mathrm{C}, \mathrm{R}_{\mathrm{f}}: 0.48$ (ethyl acetate: hexane, 3:7). IR $\left(\mathrm{cm}^{-1}\right)$ : 3,730 (O-H stretch), 2,918 (aliphatic C-H), 1,628 (C=O), 1,539 $\left(\mathrm{C}=\mathrm{C}\right.$ stretch), $1,422\left(\mathrm{C}-\mathrm{H}\right.$ bending of $\left.\mathrm{CH}_{2}\right)$, and $1,266(\mathrm{C}-\mathrm{O})$. ${ }^{1} \mathrm{HNMR}\left(400 \mathrm{MHz}, \mathrm{CDCl}_{3}\right): \delta 2.99\left(3 \mathrm{H}, \mathrm{s}, \mathrm{N}-\mathrm{CH}_{3}\right), 3.21(3 \mathrm{H}, \mathrm{s}$, $\left.\mathrm{N}-\mathrm{CH}_{3}\right), 5.64(1 \mathrm{H}, \mathrm{d}, \mathrm{J}=9.6, \mathrm{H}-2), 7.41\left(1 \mathrm{H}, \mathrm{d}, \mathrm{J}=2, \mathrm{H}-4^{\prime \prime}\right) 7.53$ $\left(1 \mathrm{H}, \mathrm{d}, \mathrm{J}=2, \mathrm{H}-6^{\prime \prime}\right)$, and $7.92(1 \mathrm{H}, \mathrm{d}, \mathrm{J}=9.6, \mathrm{H}-3) .{ }^{13} \mathrm{C} \mathrm{NMR}$ (100.6 MHz, $\left.\mathrm{CDCl}_{3}\right)$ : $190.66(\mathrm{C}-1), 164.10\left(\mathrm{C}-2^{\prime \prime}\right), 154.06$ (C-3), $134.44\left(\mathrm{C}-4^{\prime \prime}\right), 130.60\left(\mathrm{C}-6^{\prime \prime}\right), 126.63\left(\mathrm{C}-5^{\prime \prime}\right), 122.20\left(\mathrm{C}-1^{\prime \prime}\right)$, $119.67\left(\mathrm{C}-3^{\prime \prime}\right)$, and $89.93(\mathrm{C}-2)$. HR-MS for $\mathrm{C}_{11} \mathrm{H}_{11} \mathrm{Cl}_{2} \mathrm{NO}_{2}[\mathrm{M}$ $+\mathrm{H}]^{+} m / z$ : Calcd 260.0239; Observed 260.0224. The major mass fragments observed were $\mathrm{C}_{7} \mathrm{H}_{3} \mathrm{Cl}_{2} \mathrm{O}_{2}^{+}(188), \mathrm{C}_{5} \mathrm{H}_{8} \mathrm{NO}^{+}(98)$, and $\mathrm{C}_{4} \mathrm{H}_{10} \mathrm{~N}^{+}(72)$.

\section{3-Dimethylamino-1-(2-hydroxy-5-methylphenyl) propenone (3s)}

It was obtained as bright yellow solid in $84 \%$ yield; m.p.: 105-109 ${ }^{\circ} \mathrm{C}, \mathrm{R}_{\mathrm{f}}: 0.52$ (ethyl acetate: hexane, 3:7). IR $\left(\mathrm{cm}^{-1}\right)$ : 3,742 (O-H stretch), 2,922 (aliphatic $\mathrm{C}-\mathrm{H}), 1,639(\mathrm{C}=\mathrm{O}), 1,550$ $\left(\mathrm{C}=\mathrm{C}\right.$ stretch), $1,431\left(\mathrm{C}-\mathrm{H}\right.$ bending of $\left.\mathrm{CH}_{2}\right)$, and 1,253 (C-O). ${ }^{1} \mathrm{HNMR}\left(400 \mathrm{MHz}, \mathrm{CDCl}_{3}\right): \delta 2.97\left(3 \mathrm{H}, \mathrm{s}, \mathrm{N}-\mathrm{CH}_{3}\right), 3.18(3 \mathrm{H}, \mathrm{s}$, $\left.\mathrm{N}-\mathrm{CH}_{3}\right), 5.61(1 \mathrm{H}, \mathrm{d}, \mathrm{J}=9.6, \mathrm{H}-2), 6.27\left(1 \mathrm{H}, \mathrm{d}, \mathrm{J}=7.2 \mathrm{~Hz}, \mathrm{H}-3^{\prime \prime}\right)$ 7.01-7.05 $\left(1 \mathrm{H}, \mathrm{m}, \mathrm{H}-4^{\prime \prime}\right), 7.81\left(1 \mathrm{H}, \mathrm{d}, \mathrm{J}=2, \mathrm{H}-6^{\prime \prime}\right)$, and $7.89(1 \mathrm{H}$, $\mathrm{d}, \mathrm{J}=9.6, \mathrm{H}-3) .{ }^{13} \mathrm{C}$ NMR $\left(100.6 \mathrm{MHz}, \mathrm{CDCl}_{3}\right): 190.75(\mathrm{C}-1)$, 164.10 (C-2"), 154.06 (C-3), 135.20 (C-4"), 130.46 (C-6"), 129.54 (C-5"), 124.10 (C-1"), $119.12\left(\mathrm{C}-3^{\prime \prime}\right)$, and 89.93 (C-2). HR-MS for $\mathrm{C}_{12} \mathrm{H}_{15} \mathrm{NO}_{2}[\mathrm{M}+\mathrm{H}]^{+} \mathrm{m} / z$ : Calcd 206.1175; Observed 206.1169. The major mass fragments observed were $\mathrm{C}_{8} \mathrm{H}_{7} \mathrm{O}_{2}{ }^{+}(133), \mathrm{C}_{5} \mathrm{H}_{8} \mathrm{NO}^{+}(98)$, and $\mathrm{C}_{4} \mathrm{H}_{10} \mathrm{~N}^{+}(72)$.

\section{3-Dimethylamino-1-(5-fluoro-2-hydroxyphenyl) propenone (3t)}

It was obtained as pale yellow solid in $88 \%$ yield; m.p.: $140-145^{\circ} \mathrm{C}$, $\mathrm{R}_{\mathrm{f}}: 0.45$ (ethyl acetate: hexane, 3:7). IR $\left(\mathrm{cm}^{-1}\right): 3,740$ (O-H stretch), 2,928 (aliphatic $\mathrm{C}-\mathrm{H}), 1,641(\mathrm{C}=\mathrm{O}), 1,557(\mathrm{C}=\mathrm{C}$ stretch), 1,435 (C-H bending of $\mathrm{CH}_{2}$ ), and $1,258(\mathrm{C}-\mathrm{O}) .{ }^{1} \mathrm{HNMR}(400 \mathrm{MHz}$, $\left.\mathrm{CDCl}_{3}\right): \delta 2.96\left(3 \mathrm{H}, \mathrm{s}, \mathrm{N}-\mathrm{CH}_{3}\right), 3.15\left(3 \mathrm{H}, \mathrm{s}, \mathrm{N}-\mathrm{CH}_{3}\right), 5.57(1 \mathrm{H}, \mathrm{d}, \mathrm{J}$ = 9.6, H-2), $6.29\left(1 \mathrm{H}, \mathrm{d}, J=7.2 \mathrm{~Hz}, \mathrm{H}-3^{\prime \prime}\right) 7.03-7.07(1 \mathrm{H}, \mathrm{m}, \mathrm{H}-$ $\left.4^{\prime \prime}\right), 7.82\left(1 \mathrm{H}, \mathrm{d}, \mathrm{J}=2, \mathrm{H}-6{ }^{\prime \prime}\right)$, and $7.87(1 \mathrm{H}, \mathrm{d}, \mathrm{J}=9.6, \mathrm{H}-3) .{ }^{13} \mathrm{C}$ NMR (100.6 MHz, CDCl $)$ : 190.63 (C-1), $161.047\left(\mathrm{C}-5^{\prime \prime}\right), 156.13$ $\left(\mathrm{C}-2^{\prime \prime}\right), 154.32(\mathrm{C}-2), 127.05\left(\mathrm{C}-1^{\prime \prime}\right), 121.10\left(\mathrm{C}-4^{\prime \prime}\right), 117.23\left(\mathrm{C}-6^{\prime \prime}\right)$, $115.04\left(\mathrm{C}-3^{\prime}\right)$, and $89.83(\mathrm{C}-2)$. HR-MS for $\mathrm{C}_{11} \mathrm{H}_{12} \mathrm{FNO}_{2}[\mathrm{M}+$ $\mathrm{H}]^{+} \mathrm{m} / z$ : Calcd 210.0924; Observed 210.0920. The major mass fragments observed were $\mathrm{C}_{21} \mathrm{H}_{33} \mathrm{O}_{3}{ }^{+}(333), \mathrm{C}_{7} \mathrm{H}_{4} \mathrm{FO}_{2}{ }^{+}(139)$, $\mathrm{C}_{6} \mathrm{H}_{4} \mathrm{FO}^{+}(111), \mathrm{C}_{5} \mathrm{H}_{8} \mathrm{NO}^{+}(98)$, and $\mathrm{C}_{4} \mathrm{H}_{10} \mathrm{~N}^{+}(72)$.

\section{3-Dimethylamino-1-(5-chloro-2-hydroxy- 4-methyl-phenyl)propenone (3u)}

It was obtained as bright yellow solid in $80 \%$ yield; m.p.: $131-135^{\circ} \mathrm{C}, \mathrm{R}_{\mathrm{f}}$ : 0.49 (ethyl acetate: hexane, $\left.3: 7\right)$. IR $\left(\mathrm{cm}^{-1}\right)$ : 3,742 (O-H stretch), 2,922 (aliphatic $\mathrm{C}-\mathrm{H}), 1,639(\mathrm{C}=\mathrm{O}), 1,550$ $\left(\mathrm{C}=\mathrm{C}\right.$ stretch), $1,431 \quad\left(\mathrm{C}-\mathrm{H}\right.$ bending of $\left.\mathrm{CH}_{2}\right)$, and 1,253 (C-O). ${ }^{1} \mathrm{HNMR}\left(400 \mathrm{MHz}, \mathrm{CDCl}_{3}\right): \delta 2.41\left(3 \mathrm{H}, \mathrm{CH}_{3}\right), 2.96$ $\left(3 \mathrm{H}, \mathrm{s}, \mathrm{N}-\mathrm{CH}_{3}\right), 3.10\left(3 \mathrm{H}, \mathrm{s}, \mathrm{N}-\mathrm{CH}_{3}\right), 5.65(1 \mathrm{H}, \mathrm{d}, \mathrm{J}=9.6, \mathrm{H}-$ 
2), $6.38\left(1 \mathrm{H}, \mathrm{d}, \mathrm{J}=7.2 \mathrm{~Hz}, \mathrm{H}-3^{\prime \prime}\right), 7.71\left(1 \mathrm{H}, \mathrm{d}, \mathrm{J}=2, \mathrm{H}-6^{\prime \prime}\right)$, and $7.87(1 \mathrm{H}, \mathrm{d}, \mathrm{J}=9.6, \mathrm{H}-3) .{ }^{13} \mathrm{C} \mathrm{NMR}\left(100.6 \mathrm{MHz}, \mathrm{CDCl}_{3}\right): 190.71$ (C-1), 158.67 (C-2), 155.15 (C-2"), $144.33\left(\mathrm{C}-4^{\prime \prime}\right), 131.53\left(\mathrm{C}-6^{\prime \prime}\right)$, $129.31\left(\mathrm{C}-5^{\prime \prime}\right), 122.10\left(\mathrm{C}-1^{\prime}\right), 117.32\left(\mathrm{C}-3^{\prime \prime}\right)$, and $86.87(\mathrm{C}-3)$. HRMS for $\mathrm{C}_{12} \mathrm{H}_{14} \mathrm{ClNO}_{2}[\mathrm{M}+\mathrm{H}]^{+} m / z$ : Calcd 240.0785; Observed 240.0779. The major mass fragments observed were $\mathrm{C}_{8} \mathrm{H}_{7} \mathrm{ClO}_{2}^{+}(169), \mathrm{C}_{5} \mathrm{H}_{8} \mathrm{NO}^{+}(98)$, and $\mathrm{C}_{4} \mathrm{H}_{10} \mathrm{~N}^{+}(72)$.

\section{General Method for the Synthesis of 3-lodochromones (4a-4u)}

The chromones were synthesized by taking substituted 3(dimethylamino)-1-(2-hydroxyphenyl) propenone $(0.54 \mathrm{mmol})$ in $\mathrm{CHCl}_{3}(15 \mathrm{ml})$ and iodine $(1.09 \mathrm{mmol}, 2 \mathrm{~mol}$ equiv), followed by stirring the mixture at $25^{\circ} \mathrm{C}$ for $8 \mathrm{~h}$ (Scheme 1) (Gammill, 1979). The formation of products was confirmed by thin-layer chromatography (TLC) in ethyl acetate:hexane (3:7) solvent system. The solution was washed with saturated $\mathrm{Na}_{2} \mathrm{~S}_{2} \mathrm{O}_{3}$ $(15 \mathrm{ml})$, and the aqueous layer was extracted with $\mathrm{CHCl}_{3}(20 \mathrm{ml})$. The solvent was removed using Heidolph rotary evaporator HeiVAP. The synthesized chromones were purified by column chromatography to afford the pure chromones in $67-89 \%$ yield. Compounds 4f, 4g, 4h, 4i, 4j, 4k, 4l, 4m, 4n, and 40 are reported for the first time in the literature.

\section{3-lodochromone (4a)}

It was obtained as a white solid in $79 \%$ yield; m.p.: $102-103^{\circ} \mathrm{C} ; \mathrm{R}_{\mathrm{f}}$ : 0.49 (ethyl acetate: hexane, 3:7). IR $\left(\mathrm{cm}^{-1}\right)$ : 3,071 (aromatic C-H

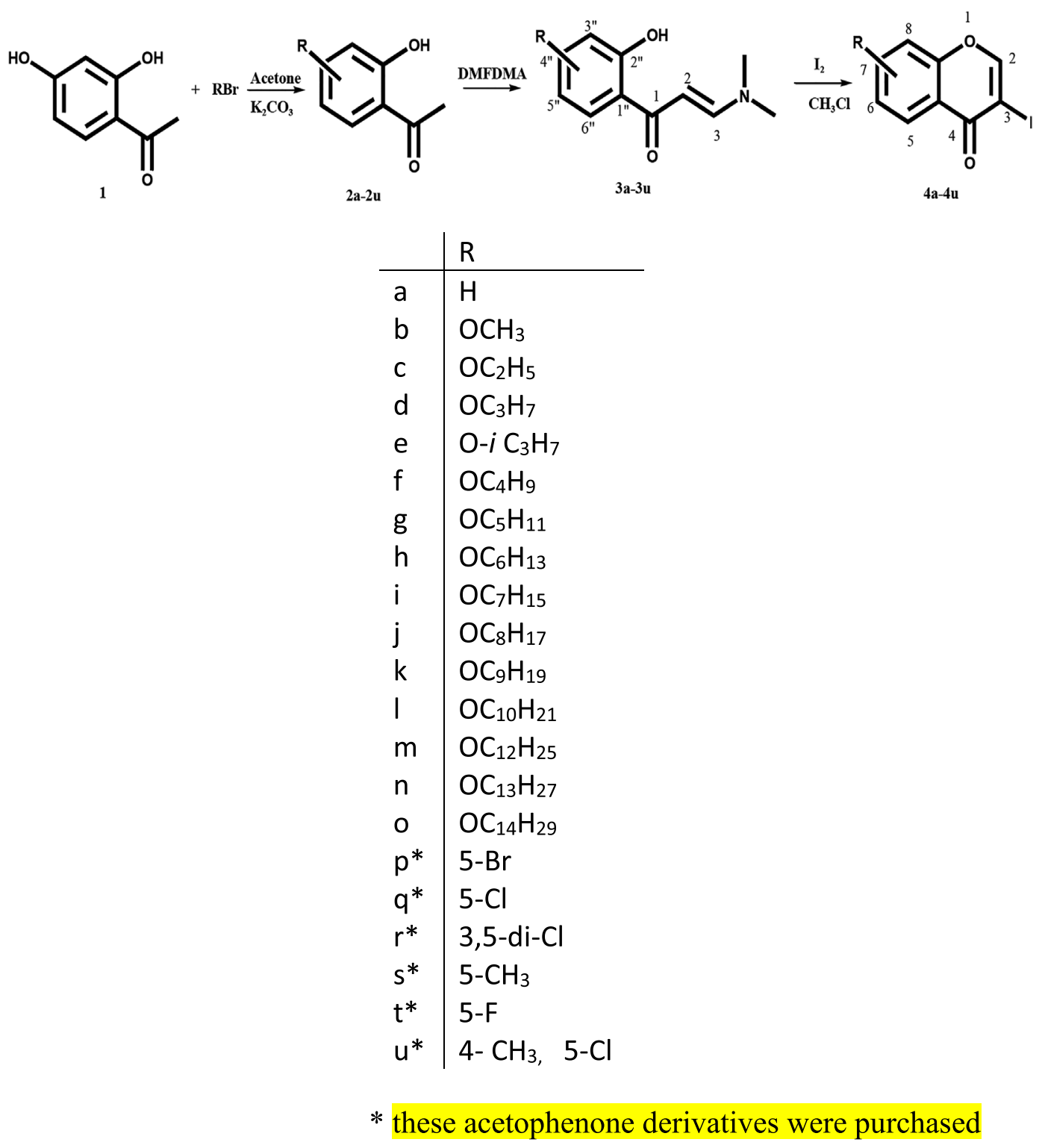

SCHEME 1 | Synthesis of 3-iodochromones (Gammill, 1979). 
stretch), 2,932 (aliphatic C-H stretch), 1,646 (C=O stretch), 1,617(aromatic $\mathrm{C}=\mathrm{C}$ stretch), 1,561 (pyrone ring $\mathrm{C}=\mathrm{C}$ stretch), 1,426 (C-H bending of $\mathrm{CH}_{2}$ ), and 1,260 (C-O Stretch). ${ }^{1} \mathrm{H} \mathrm{NMR}$ $\left(400 \mathrm{MHz}, \mathrm{CDCl}_{3}\right): \delta 6.83(1 \mathrm{H}, \mathrm{d}, \mathrm{J}=2.4 \mathrm{~Hz}, \mathrm{H}-8), 6.93-6.98(1 \mathrm{H}$, $\mathrm{m}, \mathrm{H}-6, \mathrm{H}-7), 8.08(1 \mathrm{H}, \mathrm{d}, \mathrm{J}=8.8 \mathrm{~Hz}, \mathrm{H}-5)$, and $8.17(1 \mathrm{H}, \mathrm{s}, \mathrm{H}-2)$. ${ }^{13} \mathrm{C}$ NMR (100.6 MHz, $\left.\mathrm{CDCl}_{3}\right): 175.44(\mathrm{C}-4), 158.93(\mathrm{C}-2)$, 155.99 (C-9), 134.11 (C-7), 126.32 (C-5), 123.70 (C-10), 118.30 (C-6), 101.33 (C-8), and 94.78 (C-1). HR-MS for $\mathrm{C}_{9} \mathrm{H}_{5} \mathrm{IO}_{2}[\mathrm{M}+\mathrm{H}]^{+} m / z$ : Calcd 272.9407; Observed 272.9406. The major mass fragment observed was $\mathrm{C}_{9} \mathrm{H}_{6} \mathrm{IO}_{3}{ }^{+}$(288).

\section{3-lodo-7-methoxychromone (4b)}

It was obtained as white solid in $89 \%$ yield; m.p.: $140-145^{\circ} \mathrm{C}, \mathrm{R}_{\mathrm{f}}$ : 0.51 (ethyl acetate: hexane, 3:7). IR $\left(\mathrm{cm}^{-1}\right): 3,068$ (aromatic C-H stretch), 2,934 (aliphatic C-H stretch), 1,642 (C=O stretch), 1,612 (aromatic $\mathrm{C}=\mathrm{C}$ stretch), 1,559 (pyrone ring $\mathrm{C}=\mathrm{C}$ stretch), 1,434 (C-H bending of $\mathrm{CH}_{2}$ ), and 1,258 (C-O Stretch). ${ }^{1} \mathrm{H}$ NMR $\left(400 \mathrm{MHz}, \mathrm{CDCl}_{3}\right): \delta 3.87\left(3 \mathrm{H}, \mathrm{s}, \mathrm{OCH}_{3}\right), 6.80(1 \mathrm{H}, \mathrm{d}, \mathrm{J}=$ $2.4 \mathrm{~Hz}, \mathrm{H}-8), 6.95-6.97(1 \mathrm{H}, \mathrm{m}, \mathrm{H}-6), 8.10(1 \mathrm{H}, \mathrm{d}, \mathrm{J}=8.8 \mathrm{~Hz}$, $\mathrm{H}-5)$, and $8.18(1 \mathrm{H}, \mathrm{s}, \mathrm{H}-2) .{ }^{13} \mathrm{C} \mathrm{NMR}\left(100.6 \mathrm{MHz}, \mathrm{CDCl}_{3}\right)$ : 174.72 (C-4), 164.36 (C-7), 158.03 (C-2), 157.27 (C-9), 127.71 (C-5), 117.53 (C-6), 115.11 (C-10), 100.51 (C-8), and 55.99 $\left(\mathrm{OCH}_{3}\right)$. HR-MS for $\mathrm{C}_{10} \mathrm{H}_{7} \mathrm{IO}_{3}[\mathrm{M}+\mathrm{H}]^{+} \mathrm{m} / z$ : Calcd 302.9512; Observed 302.9518. The major mass fragment observed was $\mathrm{C}_{9} \mathrm{H}_{6} \mathrm{IO}_{3}^{+}$(288).

\section{Ethoxy-3-lodochromone (4c)}

It was obtained as white solid in $82 \%$ yield; m.p.: $100-105^{\circ} \mathrm{C}, \mathrm{R}_{\mathrm{f}}$ : 0.47 (ethyl acetate: hexane, 3:7). IR $\left(\mathrm{cm}^{-1}\right.$ ): 3,065 (aromatic C-H stretch), 2,946 (aliphatic C-H stretch), 1,640 (C=O stretch), 1,617 (aromatic $\mathrm{C}=\mathrm{C}$ stretch), 1,565 (pyrone ring $\mathrm{C}=\mathrm{C}$ stretch), 1,436 (C-H bending of $\mathrm{CH}_{2}$ ), and 1,266 (C-O Stretch). ${ }^{1} \mathrm{H}$ NMR $\left(400 \mathrm{MHz}, \mathrm{CDCl}_{3}\right): \delta 1.42\left(3 \mathrm{H}, \mathrm{t}, \mathrm{J}=6.8 \mathrm{~Hz}, \mathrm{H}-2^{\prime}\right), 4.04(2 \mathrm{H}$, $\left.\mathrm{q}, \mathrm{J}=6.8 \mathrm{~Hz}, \mathrm{H}-1^{\prime}\right), 6.29(1 \mathrm{H}, \mathrm{d}, \mathrm{J}=2.4 \mathrm{~Hz}, \mathrm{H}-8), 6.34-6.36(1 \mathrm{H}$, $\mathrm{m}, \mathrm{H}-6), 7.82(1 \mathrm{H}, \mathrm{d}, \mathrm{J}=8.8 \mathrm{~Hz}, \mathrm{H}-5)$, and $7.93(1 \mathrm{H}, \mathrm{s}, \mathrm{H}-2) .{ }^{13} \mathrm{C}$ NMR (100.6 MHz, $\mathrm{CDCl}_{3}$ ): 173.13 (C-4), 165.53 (C-7), 163.56 (C-2), 162.82 (C-9), 130.79 (C-5), 113.84 (C-10), 106.64 (C-6), $101.43(\mathrm{C}-8), 89.49(\mathrm{C}-3), 63.79\left(\mathrm{C}-1^{\prime}\right)$, and $15.00\left(\mathrm{C}-2^{\prime}\right)$. HR-MS for $\mathrm{C}_{11} \mathrm{H}_{9} \mathrm{IO}_{3}[\mathrm{M}+\mathrm{H}]^{+} m / z$ : Calcd 316.9669; Observed 316.9652. The major mass fragment observed was $\mathrm{C}_{9} \mathrm{H}_{6} \mathrm{IO}_{3}{ }^{+}(288)$.

\section{3-lodo-7-propoxychromone (4d)}

It was obtained as yellowish white solid in 79\% yield; m.p.: 88-91 ${ }^{\circ} \mathrm{C}, \mathrm{R}_{\mathrm{f}}: 0.46$ (ethyl acetate: hexane, 3:7). IR $\left(\mathrm{cm}^{-1}\right): 3,071$ (aromatic C-H stretch), 2,944 (aliphatic C-H stretch), 1,642 (C=O stretch), 1,614 (aromatic $\mathrm{C}=\mathrm{C}$ stretch), 1,559 (pyrone ring $\mathrm{C}=\mathrm{C}$ stretch), 1,443 (C-H bending of $\mathrm{CH}_{2}$ ), and 1,256 (C-O Stretch). ${ }^{1} \mathrm{H}$ NMR $\left(400 \mathrm{MHz}, \mathrm{CDCl}_{3}\right): \delta 1.06\left(3 \mathrm{H}, \mathrm{t}, \mathrm{J}=7.2, \mathrm{H}-3^{\prime}\right)$, 1.73-1.88 (2H, m, H-2') $3.99\left(2 \mathrm{H}, \mathrm{t}, \mathrm{J}=6.4 \mathrm{~Hz}, \mathrm{H}-1^{\prime}\right), 6.79$ $(1 \mathrm{H}, \mathrm{d}, \mathrm{J}=2.4 \mathrm{~Hz}, \mathrm{H}-8), 6.95-6.98(1 \mathrm{H}, \mathrm{m}, \mathrm{H}-6), 8.11(1 \mathrm{H}, \mathrm{d}, \mathrm{J}=$ $9.2 \mathrm{~Hz}, \mathrm{H}-5)$, and $8.18(1 \mathrm{H}, \mathrm{s}, \mathrm{H}-2) .{ }^{13} \mathrm{C} \mathrm{NMR}(100.6 \mathrm{MHz}$, $\mathrm{CDCl}_{3}$ ): 172.56 (C-4), 164.41 (C-7), 163.56 (C-2), 162.78 (C9), 131.48 (C-5), 113.55 (C-10), 106.37 (C-6), 101.25 (C-8), 89.51 (C-3), $63.71\left(\mathrm{C}-1^{\prime}\right), 31.42\left(\mathrm{C}-2^{\prime}\right)$, and $14.98\left(\mathrm{C}-3^{\prime}\right)$. HR-MS for $\mathrm{C}_{12} \mathrm{H}_{11} \mathrm{IO}_{3}[\mathrm{M}+\mathrm{H}]^{+} m / z$ : Calcd 330.9825; Observed 330.9820 . The major mass fragment observed was $\mathrm{C}_{9} \mathrm{H}_{6} \mathrm{IO}_{3}{ }^{+}(288)$.

\section{3-lodo-7-isopropoxychromone (4e)}

It was obtained as yellowish white solid in $76 \%$ yield; m.p.: 67-70 ${ }^{\circ} \mathrm{C}, \mathrm{R}_{\mathrm{f}}: 0.50$ (ethyl acetate: hexane, 3:7). IR $\left(\mathrm{cm}^{-1}\right): 3,061$ (aromatic C-H stretch), 2,956 (aliphatic C-H stretch), 1,639 (C=O stretch), 1,612 (aromatic $\mathrm{C}=\mathrm{C}$ stretch), 1,566 (pyrone ring $\mathrm{C}=\mathrm{C}$ stretch), 1,453 (C-H bending of $\left.\mathrm{CH}_{2}\right), 1,265$ and (C-O Stretch). ${ }^{1} \mathrm{H}$ NMR (400 MHz, $\left.\mathrm{CDCl}_{3}\right): \delta 1.31\left(1 \mathrm{H}, \mathrm{d}, \mathrm{J}=4.8 \mathrm{~Hz}, \mathrm{CH}_{3}\right)$, 4.66-4.68 (1H, m, H-1' $), 6.83(1 \mathrm{H}, \mathrm{d}, \mathrm{J}=2 \mathrm{~Hz}, \mathrm{H}-8), 6.96-6.98$ $(1 \mathrm{H}, \mathrm{m}, \mathrm{H}-6), 8.38(1 \mathrm{H}, \mathrm{s}, \mathrm{H}-2), 8.15(1 \mathrm{H}, \mathrm{d}, \mathrm{J}=7.2 \mathrm{~Hz}, \mathrm{H}-5)$, and $8.22(1 \mathrm{H}, \mathrm{s}, \mathrm{H}-2) .{ }^{13} \mathrm{C}$ NMR $\left(100.6 \mathrm{MHz}, \mathrm{CDCl}_{3}\right): 172.61(\mathrm{C}-4)$, 163.82 (C-7), 157.99 (C-9), 157.32 (C-2), 127.97 (C-5), 116.32 (C6), 115.27 (C-10), 101.43 (C-8), $87.10(\mathrm{C}-3), 71.02\left(\mathrm{C}-1^{\prime}\right)$, and 21.84 (isopropyl $\mathrm{CH}_{3}$ ). HR-MS for $\mathrm{C}_{12} \mathrm{H}_{11} \mathrm{IO}_{3}[\mathrm{M}+\mathrm{H}]^{+} m / z$ : Calcd 330.9825; Observed 330.9817. The major mass fragment observed was $\mathrm{C}_{9} \mathrm{H}_{6} \mathrm{IO}_{3}{ }^{+}(288)$.

\section{7-Butoxy-3-iodochromone (4f)}

It was obtained as white solid in $81 \%$ yield; m.p.: $86-88^{\circ} \mathrm{C}, \mathrm{R}_{\mathrm{f}}: 0.57$ (ethyl acetate: hexane, 3:7). IR $\left(\mathrm{cm}^{-1}\right)$ : 3,059 (aromatic C-H stretch), 2,945 (aliphatic $\mathrm{C}-\mathrm{H}$ stretch), 1,643 ( $\mathrm{C}=\mathrm{O}$ stretch), 1,607 (aromatic $\mathrm{C}=\mathrm{C}$ stretch), 1,575 (pyrone ring $\mathrm{C}=\mathrm{C}$ stretch), 1,493 (C-H bending of $\left.\mathrm{CH}_{2}\right)$, and 1,247 (C-O Stretch). ${ }^{1} \mathrm{H}$ NMR $\left(400 \mathrm{MHz}, \mathrm{CDCl}_{3}\right): \delta 0.99(3 \mathrm{H}, \mathrm{t}, \mathrm{J}=6, \mathrm{H}-$ $\left.4^{\prime}\right), 1.47-1.81\left(4 \mathrm{H}, \mathrm{m}, \mathrm{H}-2^{\prime}\right.$ and $\left.3^{\prime}\right), 4.04\left(2 \mathrm{H}, \mathrm{t}, \mathrm{J}=5.2 \mathrm{~Hz}, \mathrm{H}-1^{\prime}\right)$, $6.80(1 \mathrm{H}, \mathrm{d}, \mathrm{J}=2 \mathrm{~Hz}, \mathrm{H}-8), 6.95-6.98(1 \mathrm{H}, \mathrm{m}, \mathrm{H}-6), 8.02(1 \mathrm{H}, \mathrm{d}, \mathrm{J}$ $=7.2 \mathrm{~Hz}, \mathrm{H}-5)$, and $8.19(1 \mathrm{H}, \mathrm{s}, \mathrm{H}-2) .{ }^{13} \mathrm{C} \mathrm{NMR}(100.6 \mathrm{MHz}$, $\mathrm{CDCl}_{3}$ ): 172.42 (C-4), 163.82 (C-7), 158.99 (C-2), 157.97 (C-9), 127.48 (C-5), 116.07 (C-6), 115.30 (C-10), 101.33 (C-8), 87.53 (C3), $68.78\left(\mathrm{C}-1^{\prime}\right), 30.95\left(\mathrm{C}-2^{\prime}\right), 19.18\left(\mathrm{C}-3^{\prime}\right)$, and $14.16\left(\mathrm{C}-4^{\prime}\right)$. HRMS for $\mathrm{C}_{13} \mathrm{H}_{13} \mathrm{IO}_{3}[\mathrm{M}+\mathrm{H}]^{+} m / z$ : Calcd 344.9982; Observed 344.9974. The major mass fragment observed was $\mathrm{C}_{9} \mathrm{H}_{6} \mathrm{IO}_{3}{ }^{+}(288)$.

\section{3-iodo-7-pentyloxychromone (4g)}

It was obtained as yellowish white solid in $86 \%$ yield; m.p.: $77-80^{\circ} \mathrm{C}, \mathrm{R}_{\mathrm{f}}: 0.52$ (ethyl acetate: hexane, $\left.3: 7\right)$. IR $\left(\mathrm{cm}^{-1}\right)$ : 3,059 (aromatic C-H stretch), 2,922 (aliphatic C-H stretch), 1,637 (C=O stretch), 1,603 (aromatic $\mathrm{C}=\mathrm{C}$ stretch), 1,570 (pyrone ring $\mathrm{C}=\mathrm{C}$ stretch), 1,471 (C-H bending of $\mathrm{CH}_{2}$ ), and 1,253 (C-O Stretch). ${ }^{1} \mathrm{H}$ NMR $\left(400 \mathrm{MHz}, \mathrm{CDCl}_{3}\right): \delta 0.93\left(3 \mathrm{H}, \mathrm{t}, \mathrm{J}=6.8, \mathrm{H}-5^{\prime}\right)$, 1.36-1.83 (6H, m, H-2', H-3', and $\left.\mathrm{H}-4^{\prime}\right), 4.02(2 \mathrm{H}, \mathrm{t}, J=$ $\left.6.4 \mathrm{~Hz}, \mathrm{H}-1^{\prime}\right), 6.79(1 \mathrm{H}, \mathrm{d}, \mathrm{J}=2.4 \mathrm{~Hz}, \mathrm{H}-8), 6.95-6.97(1 \mathrm{H}, \mathrm{m}$, $\mathrm{H}-6), 8.11(1 \mathrm{H}, \mathrm{d}, \mathrm{J}=8.8 \mathrm{~Hz}, \mathrm{H}-5)$, and $8.18(1 \mathrm{H}, \mathrm{s}, \mathrm{H}-2) .{ }^{13} \mathrm{C}$ NMR (100.6 MHz, $\mathrm{CDCl}_{3}$ ): 172.70 (C-4), 164.05 (C-7), 158.04 (C-9), 157.26 (C-2), 128.05 (C-5), 115.71 (C-6), 115.59 (C-10), $100.54(\mathrm{C}-8), 87.20(\mathrm{C}-3), 68.93\left(\mathrm{C}-1^{\prime}\right), 31.55\left(\mathrm{C}-2^{\prime}\right), 28.93\left(\mathrm{C}-3^{\prime}\right)$, $25.67\left(\mathrm{C}-4^{\prime}\right)$, and $14.19\left(\mathrm{C}-5^{\prime}\right)$. HR-MS for $\mathrm{C}_{14} \mathrm{H}_{15} \mathrm{IO}_{3}[\mathrm{M}+$ $\mathrm{H}]^{+} m / z$ : Calcd 359.0138; Observed 359.0152. The major mass fragment observed was $\mathrm{C}_{9} \mathrm{H}_{6} \mathrm{IO}_{3}{ }^{+}(288)$.

\section{7-Hexyloxy-3-iodochromone (4h)}

It was obtained as white solid in $81 \%$ yield; m.p.: $88-90^{\circ} \mathrm{C}, \mathrm{R}_{\mathrm{f}}: 0.48$ (ethyl acetate: hexane, 3:7). IR $\left(\mathrm{cm}^{-1}\right)$ : 3,063 (aromatic C-H stretch), 2,919 (aliphatic $\mathrm{C}-\mathrm{H}$ stretch), 1,635 ( $\mathrm{C}=\mathrm{O}$ stretch), 1,623 (aromatic $\mathrm{C}=\mathrm{C}$ stretch), 1,571 (pyrone ring $\mathrm{C}=\mathrm{C}$ stretch), 1,478 (C-H bending of $\left.\mathrm{CH}_{2}\right)$, and 1,257 (C-O Stretch). ${ }^{1} \mathrm{H}$ NMR (400 MHz, $\left.\mathrm{CDCl}_{3}\right): 1.42(3 \mathrm{H}, \mathrm{t}, \mathrm{J}=6.8 \mathrm{~Hz})$, 
TABLE 1 | Molecular descriptors used in the QSAR study.

\section{Descriptor}

T_2_Cl_6

T_2_F_6

T_T_F_3

DeltaEpsilonC

ZcompDipole

\section{Description}

This is the count of number of double bonded atoms (i.e. any double bonded atom, T_2) separated from chlorine atom by 6 bonds in a molecule

This is the count of number of double bonded atoms (i.e. any double bonded atom, T_2) separated from fluorine atom by 6 bonds in a molecule

Number of atoms which are separated from fluorine atom by three bonds

A measure of contribution of electronegativity

This descriptor signifies the $z$ component of the dipole moment (external coordinates)

TABLE 2 | Unicolumn statistics of training and test sets for fungicidal activity.

\begin{tabular}{|c|c|c|c|c|c|}
\hline Set & Average & Max & Min & Std. dev & Sum \\
\hline Training & -1.7971 & -0.9300 & -2.7100 & 0.4514 & -25.1600 \\
\hline Test & -1.9925 & -1.6800 & -2.5100 & 0.3648 & -7.9700 \\
\hline
\end{tabular}

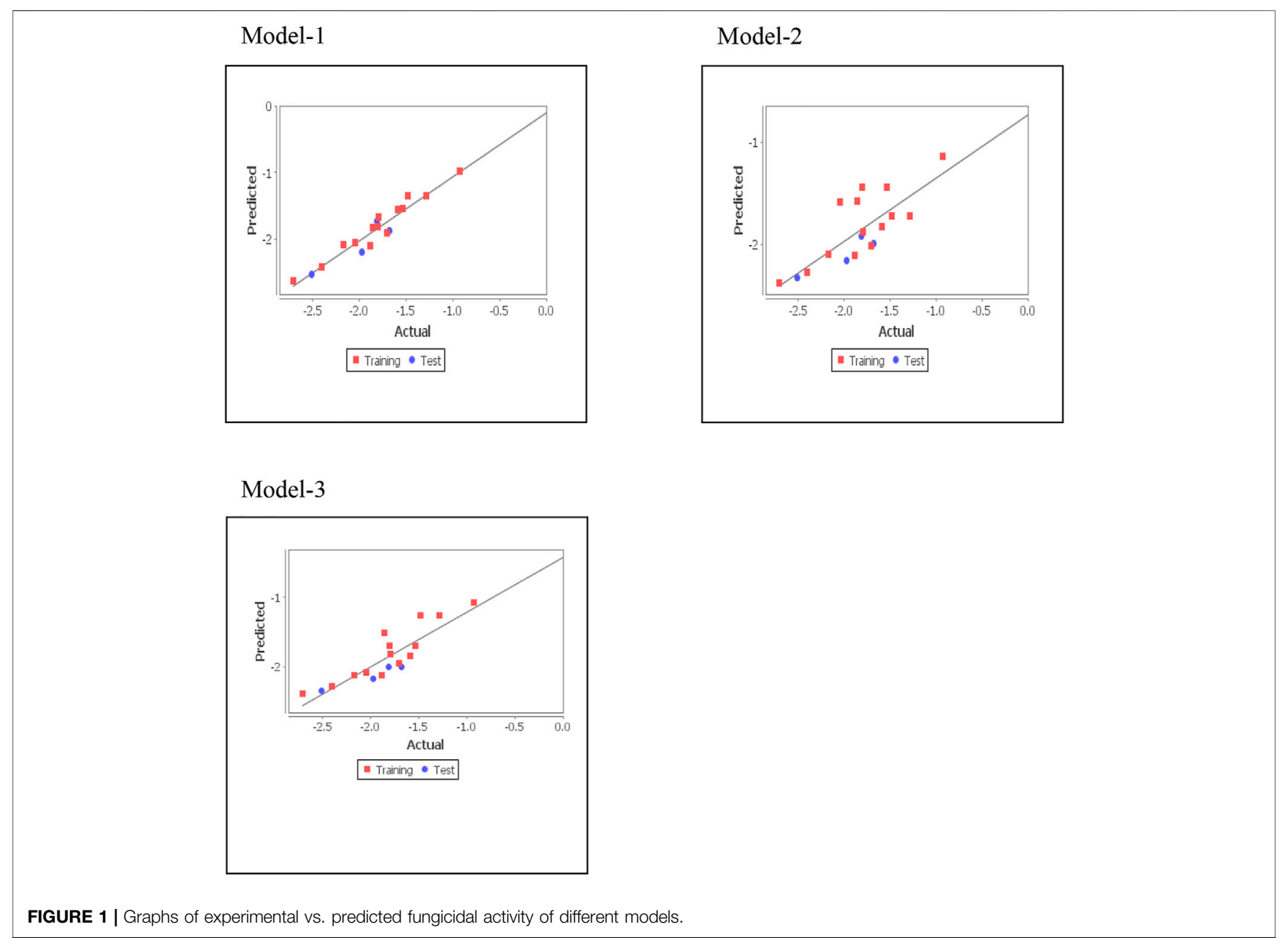

$4.06(2 \mathrm{H}, \mathrm{q}, \mathrm{J}=6.8 \mathrm{~Hz}), 6.74(1 \mathrm{H}, \mathrm{s}), 6.90-6.93(1 \mathrm{H}, \mathrm{m}), 8.05(1 \mathrm{H}$, $\mathrm{d}, \mathrm{J}=9.2 \mathrm{~Hz})$, and $8.17(1 \mathrm{H}, \mathrm{s}) .{ }^{13} \mathrm{C} \mathrm{NMR}\left(100.6 \mathrm{MHz}, \mathrm{CDCl}_{3}\right)$ : 172.70 (C-4), 163.98 (C-7), 158.03 (C-9), 157.27 (C-2), 128.04 (C-
5), 115.73 (C-6), 115.56 (C-10), 100.53 (C-8), 87.20 (C-3), 68.92 $\left(\mathrm{C}-1^{\prime}\right), 31.57\left(\mathrm{C}-2^{\prime}\right), 28.96\left(\mathrm{C}-3^{\prime}\right), 25.69\left(\mathrm{C}-4^{\prime}\right), 22.65\left(\mathrm{C}-5^{\prime}\right)$, and

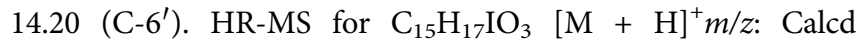


TABLE 3 | Experimental and predicted fungicidal activity of chromones.

\begin{tabular}{|c|c|c|c|c|c|c|c|c|}
\hline \multirow[t]{2}{*}{ Test compound } & \multirow[t]{2}{*}{$\mathrm{ED}_{50}\left(\mathrm{mgL}^{-1}\right)^{\mathrm{a}}$} & \multicolumn{2}{|c|}{ Fiducial limit } & \multirow[t]{2}{*}{$\chi^{2}$} & \multirow[t]{2}{*}{ Experimental ${ }_{\mathrm{p}} \mathrm{ED}_{50}{ }^{\mathrm{b}}$} & \multicolumn{3}{|c|}{ Predicted $_{\mathrm{P}} \mathrm{ED}_{50}$} \\
\hline & & Lower & Upper & & & MLR & PLS & PCR \\
\hline $4 a$ & 50.38 & 32.04 & 104.22 & 0.188 & -1.70 & -1.90 & -1.95 & -2.00 \\
\hline $4 b$ & 19.12 & 14.68 & 24.20 & 4.42 & -1.28 & -1.35 & -1.27 & -1.72 \\
\hline $4 c$ & 30.2 & 19.56 & 50.60 & 0.167 & -1.48 & -1.35 & -1.27 & -1.72 \\
\hline $4 d$ & 39.28 & 25.84 & 72.61 & 0.178 & -1.59 & -1.55 & -1.84 & -1.83 \\
\hline $4 e$ & 47.59 & 31.14 & 95.83 & 1.45 & -1.68 & -1.87 & -2.00 & -1.99 \\
\hline $4 f$ & 61.23 & 23.88 & 302.70 & 5.41 & -1.79 & -1.66 & -1.83 & -1.88 \\
\hline $4 g$ & 64.02 & 39.74 & 162.65 & 0.035 & -1.81 & -1.72 & -2.01 & -1.91 \\
\hline $4 \mathrm{~h}$ & 75.58 & 47.05 & 190.60 & 0.223 & -1.88 & -2.09 & -2.12 & -2.11 \\
\hline $4 i$ & 93.17 & 56.21 & 259.27 & 0.252 & -1.97 & -2.19 & -2.17 & -2.15 \\
\hline $4 j$ & 124.7 & 71.91 & 391.47 & 0.314 & -2.10 & -2.27 & -2.22 & -2.20 \\
\hline $4 \mathrm{k}$ & 165.7 & 91.80 & 563.30 & 0.433 & -2.22 & -2.34 & -2.25 & -2.23 \\
\hline $4 \mid$ & 248.6 & 139.68 & 742.55 & 1.724 & -2.40 & -2.41 & -2.29 & -2.27 \\
\hline $4 m$ & 325 & 173.3 & $1,116.70$ & 1.61 & -2.51 & -2.52 & -2.35 & -2.32 \\
\hline $4 n$ & 410 & 200.45 & 1811.12 & 1.134 & -2.61 & -2.57 & -2.37 & -2.35 \\
\hline 40 & 515.4 & 234.53 & 2,856.32 & 0.445 & -2.71 & -2.61 & -2.40 & -2.37 \\
\hline $4 p$ & 72.86 & 1.02 & 51.83 & 123.68 & -1.86 & -1.82 & -1.52 & -1.57 \\
\hline $4 q$ & 63.75 & 49.76 & 89.08 & 2.88 & -1.80 & -1.80 & -1.70 & -1.44 \\
\hline $4 r$ & 8.43 & 5.23 & 11.59 & 1.669 & -0.93 & -0.97 & -1.07 & -1.14 \\
\hline $4 s$ & 147.8 & 101.10 & 272.12 & 4.87 & -2.17 & -2.07 & -2.13 & -2.09 \\
\hline $4 t$ & 33.98 & 22.86 & 56.64 & 0.219 & -1.53 & -1.53 & -1.70 & -1.44 \\
\hline $4 u$ & 110.9 & 40.19 & 133.05 & 0.124 & -2.04 & -2.04 & -2.09 & -1.59 \\
\hline
\end{tabular}

${ }^{a}$ The measured in vitro fungicidal activity against $\mathrm{s}$. rolfsii

${ }^{b}$ The negative logarithm of the measured $E D_{50}\left(\mathrm{mg} \mathrm{L}^{-1}\right)$.

Mancozeb $E D_{50}=17.17 \mathrm{mg} \mathrm{L}^{-1}$.

373.0295; Observed 373.0241. The major mass fragment observed was $\mathrm{C}_{9} \mathrm{H}_{6} \mathrm{IO}_{3}{ }^{+}(288)$.

\section{7-Heptyloxy-3-iodochromone (4i)}

It was obtained as white solid in $79 \%$ yield; m.p.: $73-76^{\circ} \mathrm{C}, \mathrm{R}_{\mathrm{f}}: 0.56$ (ethyl acetate: hexane, 3:7). IR $\left(\mathrm{cm}^{-1}\right)$ : 3,083 (aromatic C-H stretch), 2,921 (aliphatic C-H stretch), 1,645 (C=O stretch), 1,612 (aromatic $\mathrm{C}=\mathrm{C}$ stretch), 1,577 (pyrone ring $\mathrm{C}=\mathrm{C}$ stretch), 1,463 (C-H bending of $\left.\mathrm{CH}_{2}\right)$, and 1,259 (C-O Stretch). ${ }^{1} \mathrm{H}$ NMR $\left(400 \mathrm{MHz}, \mathrm{CDCl}_{3}\right): \delta 0.89(3 \mathrm{H}, \mathrm{t}, \mathrm{J}=6.8$, H-7') , 1.27-1.82 (10H, m, H-2' $\left., \mathrm{H}-3^{\prime}, \mathrm{H}-4^{\prime}, \mathrm{H}-5^{\prime}, \mathrm{H}-6^{\prime}\right), 4.02(2 \mathrm{H}$, $\left.\mathrm{t}, \mathrm{J}=6.8 \mathrm{~Hz}, \mathrm{H}-1^{\prime}\right), 6.79(1 \mathrm{H}, \mathrm{d}, \mathrm{J}=2.4 \mathrm{~Hz}, \mathrm{H}-8), 6.95-6.98(1 \mathrm{H}$, $\mathrm{m}, \mathrm{H}-6), 8.12(1 \mathrm{H}, \mathrm{d}, \mathrm{J}=8.8 \mathrm{~Hz}, \mathrm{H}-5)$, and $8.18(1 \mathrm{H}, \mathrm{s}, \mathrm{H}-2) .{ }^{13} \mathrm{C}$ NMR (100.6 MHz, $\mathrm{CDCl}_{3}$ ): 172.70 (C-4), 163.99 (C-7), 158.05 (C-9), 157.27 (C-2), 128.05 (C-5), 115.72 (C-6), 115.59 (C-10), 100.54 (C-8), 87.20 (C-3), 68.93 (C-1'), 31.57-22.67 (C-2', C-3', $\left.\mathrm{C}-4^{\prime}, \mathrm{C}-5^{\prime}, \mathrm{C}-6^{\prime}\right)$, and $14.19\left(\mathrm{C}-7^{\prime}\right)$. HR-MS for $\mathrm{C}_{16} \mathrm{H}_{19} \mathrm{IO}_{3}[\mathrm{M}+$ $\mathrm{H}]^{+} m / z$ : Calcd 387.0451; Observed 387.0446. The major mass fragment observed was $\mathrm{C}_{9} \mathrm{H}_{6} \mathrm{IO}_{3}{ }^{+}(288)$.

\section{3-lodo-7-Octyloxychromone (4j)}

It was obtained as yellowish white solid in $76 \%$ yield; m.p.: $73-74^{\circ} \mathrm{C}, \mathrm{R}_{\mathrm{f}}: 0.49$ (ethyl acetate: hexane, 3:7). IR $\left(\mathrm{cm}^{-1}\right): 3,045$ (aromatic C-H stretch), 2,923 (aliphatic C-H stretch), 1,642 (C=O stretch), 1,615 (aromatic $\mathrm{C}=\mathrm{C}$ stretch), 1,593 (pyrone ring $\mathrm{C}=\mathrm{C}$ stretch), 1,468 (C-H bending of $\mathrm{CH}_{2}$ ), and 1,266 (C-O Stretch). ${ }^{1} \mathrm{H}$ NMR $\left(400 \mathrm{MHz}, \mathrm{CDCl}_{3}\right): \delta 0.87\left(3 \mathrm{H}, \mathrm{t}, \mathrm{J}=6.8, \mathrm{H}-8^{\prime}\right)$, 1.24-1.84 (12H, m, H-2', H-3', H-4 ${ }^{\prime}, \mathrm{H}-5^{\prime}, \mathrm{H}-6^{\prime}$, and $\left.\mathrm{H}-7^{\prime}\right)$, $4.03\left(2 \mathrm{H}, \mathrm{t}, \mathrm{J}=6.8 \mathrm{~Hz}, \mathrm{H}-1^{\prime}\right), 6.79(1 \mathrm{H}, \mathrm{d}, \mathrm{J}=2 \mathrm{~Hz}, \mathrm{H}-8)$, 6.95-6.98 (1H, m, H-6), $8.12(1 \mathrm{H}, \mathrm{d}, \mathrm{J}=8.8 \mathrm{~Hz}, \mathrm{H}-5)$, and $8.18(1 \mathrm{H}, \mathrm{s}, \mathrm{H}-2) .{ }^{13} \mathrm{C}$ NMR $\left(100.6 \mathrm{MHz}, \mathrm{CDCl}_{3}\right): 172.46$ (C-
4), 163.87 (C-7), 159.07 (C-2), 158.05 (C-9), 127.54 (C-5), 116.12 (C-6), 115.36 (C-10), 101.40 (C-8), 87.55 (C-3), $69.06\left(\mathrm{C}-1^{\prime}\right)$, 31.86-22.66 (C-2', C-3', C-4' $\left.{ }^{\prime}, \mathrm{C}-5^{\prime}, \mathrm{C}-6^{\prime}, \mathrm{C}-7^{\prime}\right)$, and $14.46\left(\mathrm{C}-8^{\prime}\right)$. HR-MS for $\mathrm{C}_{17} \mathrm{H}_{21} \mathrm{IO}_{3}[\mathrm{M}+\mathrm{H}]^{+} m / z$ : Calcd 401.0608; Observed 401.0601. The major mass fragment observed was $\mathrm{C}_{9} \mathrm{H}_{6} \mathrm{IO}_{3}{ }^{+}(288)$.

\section{3-lodo-7-nonyloxychromone (4k)}

It was obtained as white solid in $84 \%$ yield; m.p.: $78-80^{\circ} \mathrm{C}, \mathrm{R}_{\mathrm{f}}: 0.56$ (ethyl acetate: hexane, 3:7). IR $\left(\mathrm{cm}^{-1}\right)$ : 3,080 (aromatic C-H stretch), 2,918 (aliphatic C-H stretch), 1,636 ( $\mathrm{C}=\mathrm{O}$ stretch), 1,617 (aromatic $\mathrm{C}=\mathrm{C}$ stretch), 1,587 (pyrone ring $\mathrm{C}=\mathrm{C}$ stretch), 1,460 (C-H bending of $\left.\mathrm{CH}_{2}\right)$, and 1,264 (C-O Stretch). ${ }^{1} \mathrm{H}$ NMR $\left(400 \mathrm{MHz}, \mathrm{CDCl}_{3}\right): \delta 0.87(3 \mathrm{H}, \mathrm{t}, \mathrm{J}=6.8$, H-9 $\left.{ }^{\prime}\right), 1.26-1.82\left(14 \mathrm{H}, \mathrm{m}, \mathrm{H}-2^{\prime}, \mathrm{H}-3^{\prime}, \mathrm{H}-4^{\prime}, \mathrm{H}-5^{\prime}, \mathrm{H}-6^{\prime}, \mathrm{H}-7^{\prime}\right.$, and $\left.\mathrm{H}-8^{\prime}\right), 4.03\left(2 \mathrm{H}, \mathrm{t}, \mathrm{J}=6.4 \mathrm{~Hz}, \mathrm{H}-1^{\prime}\right), 6.79(1 \mathrm{H}, \mathrm{d}, \mathrm{J}=2 \mathrm{~Hz}, \mathrm{H}-8)$, 6.95-6.97 (1H, m, H-6), $8.11(1 \mathrm{H}, \mathrm{d}, \mathrm{J}=8.8 \mathrm{~Hz}, \mathrm{H}-5)$, and 8.18 $(1 \mathrm{H}, \mathrm{s}, \mathrm{H}-2) .{ }^{13} \mathrm{C}$ NMR $\left(100.6 \mathrm{MHz}, \mathrm{CDCl}_{3}\right): 172.70$ (C-4), 164.06 (C-7), 158.03 (C-9), 157.29 (C-2), 128.06 (C-5), 115.73 (C-6), 115.58 (C-10), 100.55 (C-8), 87.20 (C-3), $68.92 \quad\left(\mathrm{C}-1^{\prime}\right)$, 31.94-22.73 (C-3', C4', C5', C-6' $\left.{ }^{\prime}, \mathrm{C}-7^{\prime}, \mathrm{C}-8^{\prime}\right)$, and 14.19 (C$\left.9^{\prime}\right)$. HR-MS for $\mathrm{C}_{18} \mathrm{H}_{23} \mathrm{IO}_{3}[\mathrm{M}+\mathrm{H}]^{+} m / z$ : Calcd 415.0764; Observed 415.0764. The major mass fragment observed was $\mathrm{C}_{9} \mathrm{H}_{6} \mathrm{IO}_{3}{ }^{+}(288)$.

\section{7-Decyloxy-3-iodochromone (4I)}

It was obtained as cream solid in $80 \%$ yield; m.p.: $68-71^{\circ} \mathrm{C}, \mathrm{R}_{\mathrm{f}}$ : 0.46 (ethyl acetate: hexane, 3:7). IR $\left(\mathrm{cm}^{-1}\right)$ : 3,085 (aromatic C-H stretch), 2,925 (aliphatic C-H stretch), 1,641 (C=O stretch), 1,615 (aromatic $\mathrm{C}=\mathrm{C}$ stretch), 1,591 (pyrone ring $\mathrm{C}=\mathrm{C}$ stretch), 1,465 (C-H bending of $\mathrm{CH}_{2}$ ), and 1,278 (C-O Stretch). ${ }^{1} \mathrm{H}$ NMR 


\section{Model-1}

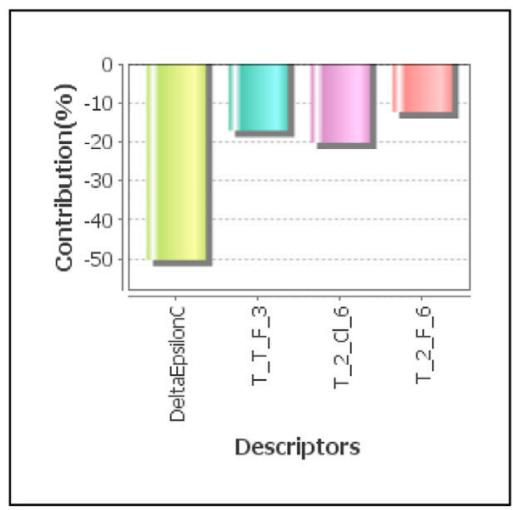

Model-3

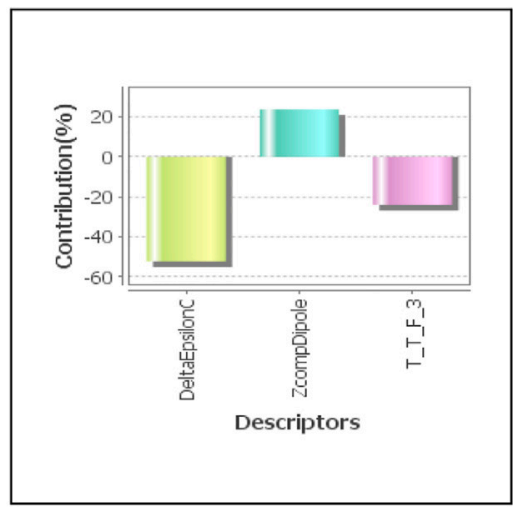

FIGURE 2 | Contribution charts of the 2D-QSAR models.

$\left(400 \mathrm{MHz}, \mathrm{CDCl}_{3}\right): \delta 0.90\left(3 \mathrm{H}, \mathrm{t}, \mathrm{J}=6.8, \mathrm{H}-10^{\prime}\right), 1.30-1.82(16 \mathrm{H}$, $\mathrm{m}, \mathrm{H}-2^{\prime}, \mathrm{H}-3^{\prime}, \mathrm{H}-4^{\prime}, \mathrm{H}-5^{\prime}, \mathrm{H}-6^{\prime}, \mathrm{H}-7^{\prime}, \mathrm{H}-8^{\prime}$ and $\left.\mathrm{H}-9^{\prime}\right), 4.02(2 \mathrm{H}, \mathrm{t}$, $\left.\mathrm{J}=6.8 \mathrm{~Hz}, \mathrm{H}-\mathrm{1}^{\prime}\right), 6.78(1 \mathrm{H}, \mathrm{d}, \mathrm{J}=2 \mathrm{~Hz}, \mathrm{H}-8), 6.94-6.97(1 \mathrm{H}, \mathrm{m}$, $\mathrm{H}-6), 8.10(1 \mathrm{H}, \mathrm{d}, \mathrm{J}=8.8 \mathrm{~Hz}, \mathrm{H}-5)$, and $8.17(1 \mathrm{H}, \mathrm{s}, \mathrm{H}-2) .{ }^{13} \mathrm{C}$ NMR (100.6 MHz, $\left.\mathrm{CDCl}_{3}\right): 172.70$ (C-4), 164.08 (C-7), 158.05 (C-9), 157.26 (C-2), 128.07 (C-5), 115.72 (C-6), 115.59 (C-10), 100.55 (C-8), 87.20 (C-3), 68.93 (C-1'), 31.96-22.75 (C-3', C4', $\left.\mathrm{C}^{\prime}, \mathrm{C}-6^{\prime}, \mathrm{C}-7^{\prime}, \mathrm{C}-8^{\prime}, \mathrm{C}-9^{\prime}\right)$, and $14.19\left(\mathrm{C}-10^{\prime}\right)$. HR-MS for $\mathrm{C}_{19} \mathrm{H}_{25} \mathrm{IO}_{3}[\mathrm{M}+\mathrm{H}]^{+} m / z$ : Calcd 429.0921; Observed 429.0946. The major mass fragment observed was $\mathrm{C}_{9} \mathrm{H}_{6} \mathrm{IO}_{3}{ }^{+}(288)$.

\section{7-Dodecyloxy-3-iodochromone (4m)}

It was obtained as white solid in $73 \%$ yield; m.p.: $58-60^{\circ} \mathrm{C}, \mathrm{R}_{\mathrm{f}}: 0.46$ (ethyl acetate: hexane, 3:7). IR $\left(\mathrm{cm}^{-1}\right): 3,082$ (aromatic C-H stretch), 2,922 (aliphatic C-H stretch), 1,639 (C=O stretch), 1,618 (aromatic $\mathrm{C}=\mathrm{C}$ stretch), 1,589 (pyrone ring $\mathrm{C}=\mathrm{C}$ stretch), 1,462 (C-H bending of $\left.\mathrm{CH}_{2}\right)$, and 1,268 (C-O Stretch). ${ }^{1} \mathrm{H}$ NMR $\left(400 \mathrm{MHz}, \mathrm{CDCl}_{3}\right): \delta 0.87(3 \mathrm{H}, \mathrm{t}, \mathrm{J}=6.8$, $\left.\mathrm{H}-12^{\prime}\right), 1.26-1.83$ (20H, m, H-2', H-3' ${ }^{\prime}, \mathrm{H}-4^{\prime}, \mathrm{H}^{-} 5^{\prime}, \mathrm{H}-6^{\prime}, \mathrm{H}-7^{\prime}, \mathrm{H}-$ $8^{\prime}, \mathrm{H}-9^{\prime}, \mathrm{H}-10^{\prime}$ and $\left.\mathrm{H}-11^{\prime}\right), 4.02\left(2 \mathrm{H}, \mathrm{t}, \mathrm{J}=6.4 \mathrm{~Hz}, \mathrm{H}-1^{\prime}\right), 6.79$ $(1 \mathrm{H}, \mathrm{d}, \mathrm{J}=2.4 \mathrm{~Hz}, \mathrm{H}-8), 6.95-6.97(1 \mathrm{H}, \mathrm{m}, \mathrm{H}-6), 8.11(1 \mathrm{H}, \mathrm{d}, \mathrm{J}=$ $9.2 \mathrm{~Hz}, \mathrm{H}-5)$, and $8.18(1 \mathrm{H}, \mathrm{s}, \mathrm{H}-2) .{ }^{13} \mathrm{C}$ NMR $(100.6 \mathrm{MHz}$, $\mathrm{CDCl}_{3}$ ): 172.75 (C-4), 163.99 (C-7), 158.03 (C-9), 157.28 (C-
Model-2

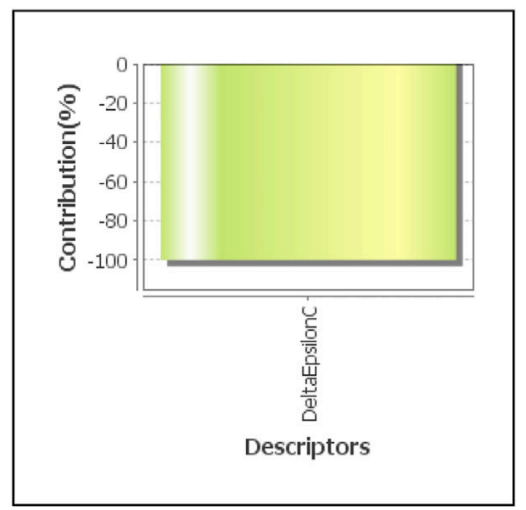

2), 128.05 (C-5), 115.73 (C-6), 115.56 (C-10), 100 (C-8), 87.24 (C3), $68.90\left(\mathrm{C}-1^{\prime}\right), 31.94-22.74\left(\mathrm{C}-3^{\prime}, \mathrm{C}^{\prime}, \mathrm{C}^{\prime}, \mathrm{C}-6^{\prime}, \mathrm{C}-7^{\prime}, \mathrm{C}-8^{\prime}, \mathrm{C}-\right.$ $\left.9^{\prime}, \mathrm{C}-10^{\prime}, \mathrm{C}-11^{\prime}\right)$, and $14.20\left(\mathrm{C}-12^{\prime}\right)$. HR-MS for $\mathrm{C}_{21} \mathrm{H}_{29} \mathrm{IO}_{3}[\mathrm{M}+$ $\mathrm{H}]^{+} m / z$ : Calcd 457.1234; Observed 457.1205. The major mass fragment observed was $\mathrm{C}_{9} \mathrm{H}_{6} \mathrm{IO}_{3}{ }^{+}(288)$.

\section{3-lodo-7-tridecyloxychromone (4n)}

It was obtained as white solid in $83 \%$ yield; m.p.: $67-70^{\circ} \mathrm{C}, \mathrm{R}_{\mathrm{f}}: 0.46$ (ethyl acetate: hexane, 3:7). IR $\left(\mathrm{cm}^{-1}\right)$ : 3,087 (aromatic C-H stretch), 2,920 (aliphatic C-H stretch), 1,643 (C=O stretch), 1,619 (aromatic $\mathrm{C}=\mathrm{C}$ stretch), 1,599 (pyrone ring $\mathrm{C}=\mathrm{C}$ stretch), 1,472 (C-H bending of $\left.\mathrm{CH}_{2}\right)$, and 1,273 (C-O Stretch). ${ }^{1} \mathrm{H}$ NMR $\left(400 \mathrm{MHz}, \mathrm{CDCl}_{3}\right): \delta 0.91(3 \mathrm{H}, \mathrm{t}, \mathrm{J}=5.6$, $\left.\mathrm{H}-13^{\prime}\right), 1.28-1.87$ (22H, m, H-2 ${ }^{\prime}, \mathrm{H}-3^{\prime}, \mathrm{H}-4^{\prime}, \mathrm{H}^{-} 5^{\prime}, \mathrm{H}-6^{\prime}, \mathrm{H}-7^{\prime}, \mathrm{H}-$ $8^{\prime}, \mathrm{H}-9^{\prime}, \mathrm{H}-10^{\prime}, \mathrm{H}-11^{\prime}$, and $\left.\mathrm{H}-12^{\prime}\right), 4.07(2 \mathrm{H}, \mathrm{t}, \mathrm{J}=5.2 \mathrm{~Hz}, \mathrm{H}-$ $\left.1^{\prime}\right), 6.84(1 \mathrm{H}, \mathrm{d}, \mathrm{J}=2 \mathrm{~Hz}, \mathrm{H}-8), 7.00-7.02(1 \mathrm{H}, \mathrm{m}, \mathrm{H}-6), 8.16(1 \mathrm{H}$, $\mathrm{d}, \mathrm{J}=7.2 \mathrm{~Hz}, \mathrm{H}-5)$, and $8.23(1 \mathrm{H}, \mathrm{s}, \mathrm{H}-2) .{ }^{13} \mathrm{C}$ NMR $(100.6 \mathrm{MHz}$, $\mathrm{CDCl}_{3}$ ): 172.62 (C-4), 163.97 (C-7), 158.00 (C-9), 157.23 (C-2), 128.00 (C-5), 115.72 (C-6), 115.53 (C-10), 100.51 (C-8), 87.21 (C3), $68.92\left(\mathrm{C}-1^{\prime}\right), 32.00-22.78\left(\mathrm{C}-3^{\prime}, \mathrm{C} 4^{\prime}, \mathrm{C} 5^{\prime}, \mathrm{C}-6^{\prime}, \mathrm{C}-7^{\prime}, \mathrm{C}-8^{\prime}, \mathrm{C}-\right.$ $\left.9^{\prime}, \mathrm{C}-10^{\prime}, \mathrm{C}-11^{\prime}, \mathrm{C}-12^{\prime}\right)$, and $14.22\left(\mathrm{C}-13^{\prime}\right)$. HR-MS for $\mathrm{C}_{22} \mathrm{H}_{31} \mathrm{IO}_{3}$ $[\mathrm{M}+\mathrm{H}]^{+} m / z$ : Calcd 457.1234; Observed 457.1257. The major mass fragment observed was $\mathrm{C}_{9} \mathrm{H}_{6} \mathrm{IO}_{3}{ }^{+}(288)$. 


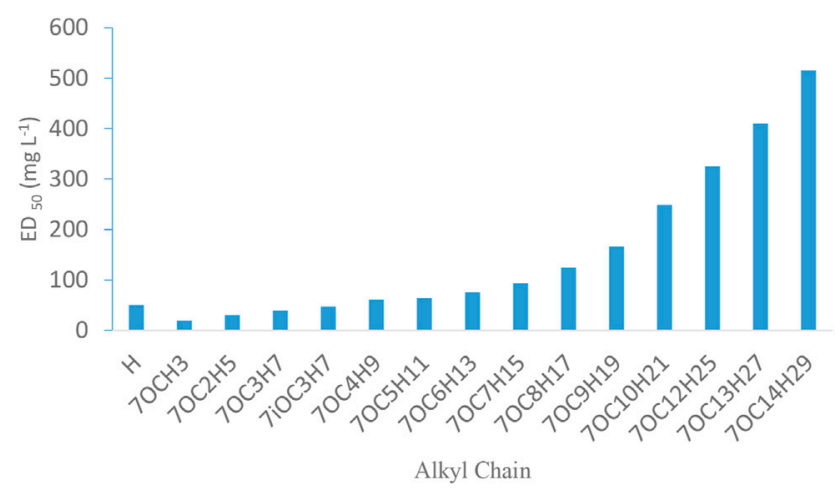

FIGURE 3 | Effect of increase in alkyl chain on fungicidal activity of chromones.

\section{3-lodo-7-tetradecyloxychromone (40)}

It was obtained as white solid in $80 \%$ yield; m.p.: $67-70^{\circ} \mathrm{C}, \mathrm{R}_{\mathrm{f}}: 0.43$ (ethyl acetate: hexane, 3:7). IR $\left(\mathrm{cm}^{-1}\right)$ : 3,081 (aromatic C-H stretch), 2,928 (aliphatic C-H stretch), 1,647 ( $\mathrm{C}=\mathrm{O}$ stretch), 1,625 (aromatic $\mathrm{C}=\mathrm{C}$ stretch), 1,588 (pyrone ring $\mathrm{C}=\mathrm{C}$ stretch), 1,469 (C-H bending of $\mathrm{CH}_{2}$ ), and 1,277 (C-O Stretch). ${ }^{1} \mathrm{H}$ NMR $\left(400 \mathrm{MHz}, \mathrm{CDCl}_{3}\right): \delta 0.90\left(3 \mathrm{H}, \mathrm{t}, \mathrm{J}=5.6, \mathrm{H}-14^{\prime}\right), 1.25-1.84(24 \mathrm{H}$, m, H-2', H-3', H-4', H-5', H-6', H-7', H-8', H-9', H-10', H-11', $\mathrm{H}-12^{\prime}$, and $\left.\mathrm{H}-13^{\prime}\right), 4.05\left(2 \mathrm{H}, \mathrm{t}, \mathrm{J}=5.2 \mathrm{~Hz}, \mathrm{H}-1^{\prime}\right), 6.85(1 \mathrm{H}, \mathrm{d}, \mathrm{J}=$ $2 \mathrm{~Hz}, \mathrm{H}-8), 6.95-6.99$ (1H, m, H-6), 8.18 (1H, d, J = 7.2 Hz, H-5), and $8.21(1 \mathrm{H}, \mathrm{s}, \mathrm{H}-2) .{ }^{13} \mathrm{C} \mathrm{NMR}\left(100.6 \mathrm{MHz}, \mathrm{CDCl}_{3}\right): 172.60(\mathrm{C}-$ 4), 164.93 (C-7), 158.04 (C-9), 157.20 (C-2), 128.04 (C-5), 115.67 (C-6), 115.50 (C-10), 100.53 (C-8), 87.20 (C-3), $68.91\left(\mathrm{C}-1^{\prime}\right)$, 32.01-22.77 (C-3', C4', C5' ${ }^{\prime}, \mathrm{C}-6^{\prime}, \mathrm{C}-7^{\prime}, \mathrm{C}-8^{\prime}, \mathrm{C}-9^{\prime}, \mathrm{C}-10^{\prime}, \mathrm{C}-11^{\prime}$, C-12', C-13'), and 14.18 (C-14'). HR-MS for $\mathrm{C}_{23} \mathrm{H}_{33} \mathrm{IO}_{3}[\mathrm{M}+$ $\mathrm{H}]^{+} m / z$ : Calcd 485.1547; Observed 485.1533. The major mass fragment observed was $\mathrm{C}_{9} \mathrm{H}_{6} \mathrm{IO}_{3}{ }^{+}(288)$.

\section{7-Bromo-3-iodochromone (4p)}

It was obtained as dark brown solid in $79 \%$ yield; m.p.: $105-110^{\circ} \mathrm{C}, \mathrm{R}_{\mathrm{f}}: 0.54$ (ethyl acetate: hexane, 3:7). IR $\left(\mathrm{cm}^{-1}\right)$ : 3,073 (aromatic C-H stretch), 2,919 (aliphatic C-H stretch), 1,636 ( $\mathrm{C}=\mathrm{O}$ stretch), 1,614 (aromatic $\mathrm{C}=\mathrm{C}$ stretch), 1,542 (pyrone ring $\mathrm{C}=\mathrm{C}$ stretch), 1,438 $\left(\mathrm{C}-\mathrm{H}\right.$ bending of $\left.\mathrm{CH}_{2}\right)$, and 1,283 (C-O Stretch). ${ }^{1} \mathrm{H}$ NMR $\left(400 \mathrm{MHz}, \mathrm{CDCl}_{3}\right): 7.41(1 \mathrm{H}, \mathrm{d}, \mathrm{J}=$ $7.2 \mathrm{~Hz}, \mathrm{H}-8), 7.80-7.82(1 \mathrm{H}, \mathrm{m}, \mathrm{H}-7), 8.32(1 \mathrm{H}, \mathrm{s}, \mathrm{H}-2)$, and 8.39 $(1 \mathrm{H}, \mathrm{d}, \mathrm{J}=2 \mathrm{~Hz}, \mathrm{H}-5) .{ }^{13} \mathrm{C} \mathrm{NMR}\left(100.6 \mathrm{MHz}, \mathrm{CDCl}_{3}\right): 172.07$ (C4), 158.11 (C-2), 155.44 (C-9), 137.74 (C-8), 132.53 (C-5), 124.43 (C-10), 120.18 (C-8), 118.17 (C-8), and 86.88 (C-3). HR-MS for $\mathrm{C}_{9} \mathrm{H}_{4} \mathrm{BrIO}_{2}[\mathrm{M}+\mathrm{H}]^{+} m / z$ : Calcd 350.8512; Observed 350.8529 . The major mass fragment observed was $\mathrm{C}_{9} \mathrm{H}_{5} \mathrm{O}_{2}{ }^{+}(145)$.

\section{6-Chloro-3-iodochromone (4q)}

It was obtained as bright yellow solid in $85 \%$ yield; m.p.: $132-136^{\circ} \mathrm{C}, \mathrm{R}_{\mathrm{f}}: 0.49$ (ethyl acetate: hexane, 3:7). IR $\left(\mathrm{cm}^{-1}\right)$ : 3,076 (aromatic C-H stretch), 2,922 (aliphatic C-H stretch), 1,639 ( $\mathrm{C}=\mathrm{O}$ stretch), 1,618 (aromatic $\mathrm{C}=\mathrm{C}$ stretch), 1,531 (pyrone ring $\mathrm{C}=\mathrm{C}$ stretch), 1,444 $\left(\mathrm{C}-\mathrm{H}\right.$ bending of $\left.\mathrm{CH}_{2}\right)$, and 1,255 (C-O Stretch). ${ }^{1} \mathrm{H}$ NMR $\left(400 \mathrm{MHz}, \mathrm{CDCl}_{3}\right): 6.93(1 \mathrm{H}, \mathrm{d}, \mathrm{J}=$
$7.2 \mathrm{~Hz}, \mathrm{H}-8), 7.15-7.27$ (1H, m, H-7), $8.32(1 \mathrm{H}, \mathrm{s}, \mathrm{H}-2)$, and 8.39 $(1 \mathrm{H}, \mathrm{d}, \mathrm{J}=2 \mathrm{~Hz}, \mathrm{H}-5) .{ }^{13} \mathrm{C} \mathrm{NMR}\left(100.6 \mathrm{MHz}, \mathrm{CDCl}_{3}\right): 172.37(\mathrm{C}-$ 4), 159.73 (C-2), 154.51 (C-9), 134.75 (C-7), 130.64 (C-6), 124.63 (C-5), 122.25 (C-10), 121.21 (C-8), and 86.69 (C-3). HR-MS for $\mathrm{C}_{9} \mathrm{H}_{4} \mathrm{ClIO}_{2}[\mathrm{M}+\mathrm{H}]^{+} \mathrm{m} / z$ : Calcd 306.9170; Observed 306.9163. The major mass fragment observed was $\mathrm{C}_{9} \mathrm{H}_{5} \mathrm{O}_{2}{ }^{+}(145)$.

\section{6, 8-Dichloro-3-iodochromone (4r)}

It was obtained as cream solid in $81 \%$ yield; m.p.: $143-145^{\circ} \mathrm{C}, \mathrm{R}_{\mathrm{f}} 0.61$ (ethyl acetate: hexane, 3:7). IR $\left(\mathrm{cm}^{-1}\right): 3,051$ (aromatic $\mathrm{C}-\mathrm{H}$ stretch), 2,923 (aliphatic C-H stretch), 1,641 ( $\mathrm{C}=\mathrm{O}$ stretch), 1,615 (aromatic $\mathrm{C}=\mathrm{C}$ stretch), 1,538 (pyrone ring $\mathrm{C}=\mathrm{C}$ stretch), 1,421 (C-H bending of $\mathrm{CH}_{2}$ ), and 1,274 (C-O Stretch). ${ }^{1} \mathrm{H}$ NMR (400 MHz, $\left.\mathrm{CDCl}_{3}\right): \delta$ $7.78(1 \mathrm{H}, \mathrm{d}, \mathrm{J}=2 \mathrm{~Hz}, \mathrm{H}-5), 8.14(1 \mathrm{H}, \mathrm{d}, \mathrm{J}=2 \mathrm{~Hz}, \mathrm{H}-7)$, and $8.38(1 \mathrm{H}$, s, H-2). ${ }^{13} \mathrm{C}$ NMR (100.6 MHz, $\left.\mathrm{CDCl}_{3}\right): 171.83$ (C-4), 157.70 (C-2), 150.63 (C-9), 134.41 (C-7), 131.64 (C-6), 124.69 (C-5), 124.43 (C10), 123.26 (C-8), and 86.91 (C-3). HR-MS for $\mathrm{C}_{9} \mathrm{H}_{3} \mathrm{Cl}_{2} \mathrm{IO}_{2}[\mathrm{M}+$ $\mathrm{H}]^{+} \mathrm{m} / z$ : Calcd 340.8627; Observed 340.8614. The major mass fragment observed was $\mathrm{C}_{9} \mathrm{H}_{5} \mathrm{O}_{2}^{+}(145)$.

\section{3-lodo-6-methylchromone (4s)}

It was obtained as yellow solid in $67 \%$ yield; m.p.: $110-116^{\circ} \mathrm{C}, \mathrm{R}_{\mathrm{f}}$ : 0.55 (ethyl acetate: hexane, 3:7). IR $\left(\mathrm{cm}^{-1}\right)$ : 3,075 (aromatic C-H stretch), 2,921 (aliphatic C-H stretch), 1,642 (C=O stretch), 1,618 (aromatic $\mathrm{C}=\mathrm{C}$ stretch), 1,540 (pyrone ring $\mathrm{C}=\mathrm{C}$ stretch), 1,453 (C-H bending of $\mathrm{CH}_{2}$ ), and 1,267 (C-O Stretch). ${ }^{1} \mathrm{H}$ NMR $\left(400 \mathrm{MHz}, \mathrm{CDCl}_{3}\right): 2.41\left(3 \mathrm{H}, \mathrm{CH}_{3}\right), 6.28(1 \mathrm{H}, \mathrm{d}, \mathrm{J}=6 \mathrm{~Hz}, \mathrm{H}-8)$, $7.32(1 \mathrm{H}, \mathrm{d}, \mathrm{J}=8.8 \mathrm{~Hz}, \mathrm{H}-7), 7.81(1 \mathrm{H}, \mathrm{d}, \mathrm{J}=2 \mathrm{~Hz}, \mathrm{H}-5)$, and 8.22 $(1 \mathrm{H}, \mathrm{s}, \mathrm{H}-2) .{ }^{13} \mathrm{C}$ NMR $\left(100.6 \mathrm{MHz}, \mathrm{CDCl}_{3}\right): 173.44$ (C-4), 157.72 (C-2), 154.46 (C-9), 136.13 (C-6), 135.45 (C-7), 125.82 (C-5), 121.49 (C-10), 117.81 (C-8), $86.72(\mathrm{C}-3)$, and $21.09\left(\mathrm{PhCH}_{3}\right)$. HR-MS for $\mathrm{C}_{10} \mathrm{H}_{7} \mathrm{IO}_{2}[\mathrm{M}+\mathrm{H}]^{+} m / z$ : Calcd 286.9563; Observed 286.9549. The major mass fragment observed was $\mathrm{C}_{10} \mathrm{H}_{7} \mathrm{O}_{2}{ }^{+}(159)$.

\section{6-Fluoro-3-iodochromone (4t)}

It was obtained as light yellow solid in $81 \%$ yield; m.p.: $123-126^{\circ} \mathrm{C}, \mathrm{R}_{\mathrm{f}}: 0.57$ (ethyl acetate: hexane, $\left.3: 7\right)$. IR $\left(\mathrm{cm}^{-1}\right)$ : 3,063 (aromatic C-H stretch), 2,942 (aliphatic C-H stretch), 1,647 ( $\mathrm{C}=\mathrm{O}$ stretch), 1,616 (aromatic $\mathrm{C}=\mathrm{C}$ stretch), 1,553 
(pyrone ring $\mathrm{C}=\mathrm{C}$ stretch), 1,436 $\left(\mathrm{C}-\mathrm{H}\right.$ bending of $\left.\mathrm{CH}_{2}\right)$, and 1,257 (C-O Stretch). ${ }^{1} \mathrm{H}$ NMR $\left(400 \mathrm{MHz}, \mathrm{CDCl}_{3}\right): 7.41(1 \mathrm{H}, \mathrm{d}, \mathrm{J}=$ $7.2 \mathrm{~Hz}, \mathrm{H}-8), 7.80-7.82(1 \mathrm{H}, \mathrm{m}, \mathrm{H}-7), 8.32(1 \mathrm{H}, \mathrm{s}, \mathrm{H}-2)$, and 8.39 $(1 \mathrm{H}, \mathrm{d}, \mathrm{J}=2 \mathrm{~Hz}, \mathrm{H}-5) .{ }^{13} \mathrm{C} \mathrm{NMR}\left(100.6 \mathrm{MHz}, \mathrm{CDCl}_{3}\right): 172.77$ (C4), 161.04 (C-6), 158.01 (C-2), 152.45 (C-9), 122.84 (C-7), 120.46 (C-8), 111.52 (C-5), and $86.69(\mathrm{C}-3)$. HR-MS for $\mathrm{C}_{9} \mathrm{H}_{4} \mathrm{ClIO}_{2}$ $[\mathrm{M}+\mathrm{H}]^{+} m / z$ : Calcd 290.9262; Observed 290.9258. The major mass fragment observed was $\mathrm{C}_{9} \mathrm{H}_{5} \mathrm{O}_{2}{ }^{+}(145)$.

\section{6-Chloro-3-iodo-7-methylchromone (4u)}

It was obtained as light yellow solid in $83 \%$ yield; m.p.: $120-125^{\circ} \mathrm{C}$, $\mathrm{R}_{\mathrm{f}} 0.50$ (ethyl acetate: hexane, 3:7). IR $\left(\mathrm{cm}^{-1}\right): 3,067$ (aromatic C-H stretch), 2,918 (aliphatic C-H stretch), 1,638 (C=O stretch), 1,612 (aromatic $\mathrm{C}=\mathrm{C}$ stretch), 1,538 (pyrone ring $\mathrm{C}=\mathrm{C}$ stretch), and 1,259 (C-O Stretch). ${ }^{1} \mathrm{H}$ NMR (400 MHz, $\left.\mathrm{CDCl}_{3}\right): 2.47\left(3 \mathrm{H}, \mathrm{CH}_{3}\right)$, $7.33(1 \mathrm{H}, \mathrm{s}, \mathrm{H}-8), 8.15(1 \mathrm{H}, \mathrm{s}, \mathrm{H}-5)$, and $8.23(1 \mathrm{H}, \mathrm{s}, \mathrm{H}-2) .{ }^{13} \mathrm{C} \mathrm{NMR}$ $\left(100.6 \mathrm{MHz}, \mathrm{CDCl}_{3}\right): 172.37$ (C-4), 159.73 (C-2), 154.51 (C-9), 145.53 (C-7), 131.33 (C-5), 129.12 (C-6), 123.01 (C-10), 120.13 (C8 ), and 86.59 (C-3). HR-MS for $\mathrm{C}_{10} \mathrm{H}_{6} \mathrm{ClIO}_{2}[\mathrm{M}+\mathrm{H}]^{+} m / z$ : Calcd 320.9173; Observed 320.9158. The major mass fragment observed was $\mathrm{C}_{10} \mathrm{H}_{7} \mathrm{O}_{2}{ }^{+}(159)$.

\section{Bioefficacy \\ Test Fungus}

The test fungus S. rolfsii ITCC 6474 was procured from Indian Type Culture Collection (ITCC) center, Division of Plant Pathology, ICAR-Indian Agricultural Research Institute, New Delhi-110012, India and kept at $27^{\circ} \mathrm{C}$ for at least $4-7$ days on Potato Dextrose Agar (PDA) slant. The fungus was subcultured in Petri plates for further bioassay studies.

\section{In vitro Fungicidal Activity}

A stock solution $\left(1,000 \mathrm{mgL}^{-1}\right)$ of each synthesized compound was prepared in DMSO. Preliminary screening was carried out at different concentrations. A final bioassay was conducted at five different concentrations namely $1,000,500,250,125$, and $62.50 \mathrm{mg} \mathrm{L}^{-1}$ of $4 \mathrm{i}-4 \mathrm{n}, 4 \mathrm{p}, 4 \mathrm{~s}$, and $4 \mathrm{u}$ chromones, and all other chromones were tested at 100, 50, 25, 12.5, and $6.25 \mathrm{mg} \mathrm{L}^{-1}$, respectively. All concentrations were tested in triplicates. Commercially available fungicide Mancozeb (technical) was taken as positive control.

An in vitro antifungal bioassay was carried out on PDA medium by poisoned food technique (Nene and Thapliyal, 1979). Fungal growth (colony diameter) was measured and percentage inhibition was calculated by Abbott's formula (Abbott, 1925).

$$
\text { Percentage inhibition }(\mathrm{I})=(\mathrm{C}-\mathrm{T}) \times 100 / \mathrm{C},
$$

where $\mathrm{C}=$ colony diameter $(\mathrm{mm})$ of the control and $\mathrm{T}=$ colony diameter $(\mathrm{mm})$ of the test plate.

Corrected percentage inhibition (IC) was calculated by given formula.

$$
I C=(\mathrm{I}-\mathrm{CF}) /(100-\mathrm{CF}) \times 100,
$$

where $\mathrm{I}=$ Percentage inhibition, $\mathrm{CF}=(90-\mathrm{C}) / \mathrm{C} \times 100,90$ is the diameter $(\mathrm{mm})$ of the Petri plate, and $\mathrm{C}$ is the growth of the fungus $(\mathrm{mm})$ in control.
$\mathrm{ED}_{50}\left(\mathrm{mg} \mathrm{L}^{-1}\right)$ values (Effective Dose for $50 \%$ inhibition) were calculated using SPSS statistical Package (v16.0).

\section{Quantitative Structure Activity Relationship}

QSAR analysis was done by taking negative logarithm of observed $\mathrm{ED}_{50}\left(\mathrm{mgL}^{-1}\right)\left[\mathrm{pED}_{50}=-\log \left(\mathrm{ED}_{50}\right)\right]$ as dependent variable and 2D descriptors (Table 1) as independent variables. 2D Structures of compounds were drawn in Chemdraw Ultra 7.0 software and converted to $3 \mathrm{D}$ structures. A total of 239 2D descriptors were determined encoding different molecular structural characteristics consisting of electronic, spatial, thermodynamic, and structural descriptors, for example, element count, atomic valence connectivity index (chiV), path cluster, estate number, retention index (chi), chain path count, $\log \mathrm{P}$, semi-empirical path count, molecular cluster, molecular weight, topological index, and refractivity. Descriptors were calculated by geometry optimization and energy minimization carried out by the batch energy minimization method in the Merck molecular force field (MMFF) at RMS gradient (criteria for convergence) 0.01 , distance dependent dielectric 1 , and the number of cycles $(\max ) 1$, 000. Different Baumann alignment-independent (AI) descriptors were also calculated. All computational work was carried out with the help of VLifeMDS QSAR plus 4.6 software using the Lenovo PC with Windows 8.1 operating system and the Intel $(\mathrm{R})$ Celeron $(\mathrm{R})$ processor.

\section{Training and Test Set}

The entire data of 21 compounds were divided into a training set (14 compounds) and a test set (4 compounds), and 3 compounds were taken for validation with the help of the sphere exclusion method (Hudson et al., 1996). Unicolumn statistics were used to check the accuracy of selection of training and test sets, as the maximum value of the training set was greater than that of the test set and the minimum value of the training set was less than that of the test set (Table 2).

\section{Regression Analysis}

Regression analysis was done with three model building methods, MLR, PCR, and PLS. Various 2D descriptors were taken as independent variables and $\mathrm{pED}_{50}$ values as dependent variables by taking cross-correlation limit as 0.5 ; five variables in the final equation and $\mathrm{r}^{2}$ as the term selection criteria, F-test "in" at 4 and "out" at 3.99, $\mathrm{r}^{2}$ and F-test value. Values were fixed at 0 for variance cutoff, 10 for random iterations, and auto scaling for scaling. Developed QSAR models were assessed with the help of statistical parameters such as $n=$ total number of compounds used in regression, $\mathrm{k}=$ total number of descriptors in a model, $\mathrm{r}^{2}=$ regression coefficient, $\mathrm{q}^{2}=$ cross-validated correlation coefficient, $\mathrm{F}=\mathrm{F}$-test (Fisher test value) for statistical significance, pred_ $\mathrm{r}^{2}=$ predictive squared correlation coefficients, pred_r $^{2}$ se $=$ coefficient of correlation of predicted data set, and $\mathrm{r}^{2}$ se and $\mathrm{q}^{2} \mathrm{se}=$ standard error (SE) of estimation.

\section{Multiple Linear Regression Analysis}

MLR defines linear relationship between a single response variable and a number of predictor variables. In the present work, $\mathrm{pED}_{50}$ fungicidal activity was response variable and $2 \mathrm{D}$ descriptors were predictor variables. In this method, regression 
coefficients values $\left(\mathrm{r}^{2}\right)$ were calculated by the least squares curve fitting method. In regression analysis, conditional mean of the dependent variable $\left(\mathrm{pED}_{50}\right) \mathrm{Y}$ depends on (descriptors) X (Eq. 1).

$$
Y=b 1 \times 1+b 2 \times 2+b 3 \times 31+c
$$

where $\mathrm{Y}=$ dependent variable, " $\mathrm{x}$ "s = independent variables, " $\mathrm{b}$ "s $=$ regression coefficients for " $\mathrm{x}$ "s, and " $\mathrm{c}$ " = regression constant or intercept (Devillers, 1996; Croux and Joossens, 2005).

\section{Principal Component Regression Method}

In this method, the whole data were divided into principal components (PCs), smaller sets having major details of the large set. The main aim of PCR is to find out the values of a dependent variable with the help of selected PCs of independent variables. These PCs were not correlated, but had a basic linear relationship of original variables. The data were rotated into a new set of axes in such a way that first few axes showed greatest variability within the data. First PC (PC1) had maximum possible variation in the data, and each successive PC was taken perpendicular to preceding PCs and represent highest of the outstanding variance. The PC value is calculated by rotation of each point to a particular axis. A new group of axes for the data was chosen on the basis of a descending value data variance. Principal component analysis (PCA) also describes the fashion of similarity of the observations and the variables by exhibiting them as points in maps. PCR gives a mechanism for obtaining structure in datasets.

\section{Partial Least Squares Regression Method}

The partial least squares (PLS) test correlation between a set of dependent variables (Y) and a set of predictor variables (X). The main aim of PLS regression is helpful in describing the common structure by estimating the biological activity (dependent variables Y) from descriptors (X) (Huberty, 1994). PLS developed orthogonal components based on the relationship between predictors and respective outputs, while retaining highest variance of independent variables.

\section{Validation of The QSAR Model}

The QSAR model was validated with Leave-one-out (LOO) cross validation, by dividing training dataset into equal size subsets after eliminating one biological activity data (number of subsets $=$ number of data points). These subsets were used to develop the model for calculating predicted activity (value of response variable of excluded data). Since in LOO subset all the data points were serially considered as predicted, the mean value of predicted activity was similar for LOO $\mathrm{q}^{2}$ and $\mathrm{r}^{2}$. After elimination of the next data point, the same procedure was repeated until all data points were removed. Thus, three statistically significant models were developed by LOO crossvalidation. (Kubyani, 1994). Eq. 2 was used for calculating $q^{2}$.

$$
\mathrm{q}^{2}=1-\sum\left(\mathrm{Y}_{\text {pred }}-\mathrm{Y}_{\text {act }}\right)^{2} / \sum\left(\mathrm{Y}_{\text {act }}-\mathrm{Y}_{\text {mean }}\right)
$$

where $\mathrm{Y}_{\text {pred }}=$ predicted, $\mathrm{Y}_{\text {act }}=$ actual, $\mathrm{Y}_{\text {mean }}=$ mean values of the $\mathrm{pED}_{50}$, and $\Sigma\left(\mathrm{Y}_{\text {pred }}-\mathrm{Y}_{\mathrm{act}}\right)^{2}=$ predictive residual error sum of squares (PRESS). External validation has also been performed to verify model validity, which tests how well the equation generalizes. A training set was used to develop an adjustment model for predicting activities of test set members. The predictive performance of equations was determined by $\mathrm{q}^{2}$, and coefficients of predictive squared correlation (pred_r $\mathrm{r}^{2}$ ). pred_r $\mathrm{r}^{2}$ was calculated by Eq. 3 .

$$
\text { pred_r } \mathrm{r}^{2}=1-\sum\left(\mathrm{Y}_{\text {pred(Test) }}-\mathrm{Y}_{(\text {Test })}\right)^{2} / \sum\left(\mathrm{Y}_{\text {Test }}-\mathrm{Y}_{\text {Training }}\right)
$$

where $\mathrm{Y}_{\text {pred(Test) }}=$ predicted activity and $\mathrm{Y}_{\text {Test }}=$ observed activity of test set compounds and $\mathrm{Y}_{\text {Training }}=$ mean activity value of the training set. Statistical significance of model was validated by the fitness plot (Figure 1) and it was also supported by closeness of observed and predicted activity (Table 3 ). The magnitude of different descriptors employed for developing QSAR models were present in contribution charts (Figure 2).

\section{RESULT AND DISCUSSION}

\section{Synthesis and Characterization}

In this study, total 21 compounds $(4 a-4 u)$ were synthesized out of which 10 compounds (4f, $4 \mathrm{~g}, 4 \mathrm{~h}, \mathbf{4 i}, \mathbf{4 j}, \mathbf{4 k}, \mathbf{4 l}, \mathbf{4 m}, \mathbf{4 n}$, and $4 \mathrm{o}$ ) were novel. The compounds synthesized by the above method were obtained in the yield ranging from 67 to $89 \%$. Characterization of compounds was done by IR, ${ }^{1} \mathrm{H} N M R{ }^{13} \mathrm{C} N M R$, and LC-HRMS. In enaminones $(3 \mathrm{a}-3 \mathrm{u})$, peaks at $\delta 5.57-5.73(1 \mathrm{H}, \mathrm{d}, \mathrm{J}=8.8 \mathrm{~Hz}, \mathrm{H}-2)$ and $\delta 7.55-7.92(1 \mathrm{H}, \mathrm{d}, \mathrm{J}=8.8 \mathrm{~Hz}, \mathrm{H}-3)$ as two doublets for two protons with $\mathrm{J}=8.8 \mathrm{~Hz}$ each were representative peaks of olefinic bond in ${ }^{1} \mathrm{H}$-NMR spectrum of all the compounds, and confirms the formation of enaminone. In ${ }^{13} \mathrm{C}-\mathrm{NMR}$, the peaks at $\delta 89.75-89.93$ $(\mathrm{C}-2)$ and 154.01-158.67 (C-3) for $\mathrm{HC}=\mathrm{CH}$ and at $\delta$ 190.31-190.90 for $\mathrm{C}=\mathrm{O}$ were conspicuous for all the compounds. The higher chemical shifts values of $\mathrm{H}-3$ and $\mathrm{C}-3$ than $\mathrm{H}-2$ and $\mathrm{C}-2$ were due to carbonyl moiety, which polarizes the $\mathrm{C}=\mathrm{C}$ double bond. In IR spectra, stretching of $(\mathrm{C}=\mathrm{O})$ at 1,628-1,647 and $(\mathrm{C}=\mathrm{C})$ at $1,539-1,593 \mathrm{~cm}^{-1}$ supported the NMR data.

In case of chromones $(4 \mathrm{a}-4 \mathrm{u})$, peaks at $\delta 7.93-8.38(1 \mathrm{H}, \mathrm{s}, \mathrm{H}$ 2) in ${ }^{1} \mathrm{H}$ NMR and 157.20-163.56 (C-2) and 171.83-175.44 (C-4, $\mathrm{C}=\mathrm{O})$ in ${ }^{13} \mathrm{C}$ NMR spectra of all compounds confirms synthesis of chromone derivatives. Stretching of $(\mathrm{C}=\mathrm{O})$ at 1,635-1,647 and (pyrone ring, $\mathrm{C}=\mathrm{C}$ ) at $1,531-1,599 \mathrm{~cm}^{-1}$ in IR spectra justified the NMR data.

\section{In Vitro Fungicidal Activity}

In vitro evaluation showed that all the tested compounds $(4 \mathrm{a}-4 \mathrm{u})$ exhibited promising fungicidal activity against $S$. rolfsii (Table 3), and compound $4 r$ was found to be most active $\left(\mathrm{ED}_{50}=8.43 \mathrm{mgL}^{-1}\right)$ which was better than Mancozeb $\left(\mathrm{ED}_{50}=17.17 \mathrm{mgL}^{-1}\right)$, a commercial fungicide. It was observed that with increasing length of alkyl chain, fungicidal activity of alkoxy chromones $(4 a-4 n)$ significantly decreased (Figure 3). Among halo chromone derivatives (4p, 4q, 4r, 4t, and 4u) 6, 8 dichlro derivative (4r) exhibited the highest and 4 fluoro derivative $(\mathbf{4 u})$ the least activity.

\section{D-QSAR Study}

Three statistically significant QSAR models viz. Model-1 (MLR) Eq. 4, Model-2 (PCR) Eq. 5, and Model-3 (PLS) Eq. 6 were developed in 2D-QSAR study of fungicidal activity. 
Model-1 (MLR)

$$
\begin{aligned}
\mathrm{pEd}_{50}= & -25.5825(\text { DeltaEpsilonC })-0.2256\left(\mathrm{~T} \_\mathrm{T} \_\mathrm{F} \_3\right) \\
& -0.7476(\mathrm{~T} \text { _2_Cl_6 })-0.2716(\mathrm{~T} \text { _2_F_6 })-3.5771
\end{aligned}
$$

where $n=14, \mathrm{DF}=9, \mathrm{r}^{2}=0.9434, \mathrm{q}^{2}=0.9117$, F_test $=37.5361, \mathrm{r}^{2} \_\mathrm{se}=$ $0.1290, \mathrm{q}^{2} \_$se $=0.1612$, pred_r $\mathrm{r}^{2}=0.8374$, and pred_r $\mathrm{r}^{2} \mathrm{se}=0.1730$.

Model-2 (PCR)

$$
p E d_{50}=-13.2406(\text { DeltaEpsilonC })-2.8722
$$

where $n=14, \mathrm{DF}=12, \mathrm{r}^{2}=0.5979, \mathrm{q}^{2}=0.4509, \mathrm{~F} \_$test $=17.8453$, $\mathrm{r}^{2} \_\mathrm{se}=0.2979, \mathrm{q}^{2} \_\mathrm{se}=0.3482$, pred_r ${ }^{2}=0.6877$, and pred_r ${ }^{2}$ se $=$ 0.2397 .

\section{Model-3 (PLS)}

$$
\begin{aligned}
p E d_{50}= & 13.5036(\text { DeltaEpsilonC })+130.1390(\text { ZcompDipole }) \\
& -0.1607\left(\mathrm{~T}_{-} \mathrm{T}_{-} \mathrm{F}_{-} 3\right)-2.8393
\end{aligned}
$$

where $n=14, \mathrm{DF}=11, \mathrm{r}^{2}=0.8006, \mathrm{q}^{2}=0.6167, \mathrm{~F} \_$test $=22.0866, \mathrm{r}^{2} \_\mathrm{se}=$ $0.2191, \mathrm{q}^{2} \_\mathrm{se}=0.3038$, pred_r${ }^{2}=0.6186$, and pred_r $\mathrm{r}^{2} \mathrm{se}=0.2649$.

In above QSAR models, correlation coefficient $\left(\mathrm{r}^{2}\right)$ was used to calculate biological activity variance by multiplying with 100 . The predictive ability $\left(\mathrm{q}^{2}\right)$ of generated QSAR models was assessed by LOO (Left-out-one) method. $F$ is the ratio of variance of models and that of error in regression. Models with a higher $F$ value and lower SE of estimation $\left(\mathrm{r}^{2} \mathrm{se}\right.$ and $\left.\mathrm{q}^{2} \mathrm{se}\right)$ were considered statistically significant. External validation with pred_r $r^{2}>0.3$, established the predictive power of the QSAR model. Among these three models, the MLR model was found best as revealed by $\mathrm{q}^{2}, \mathrm{r}^{2}$, higher values of F-test, and pred_r $r^{2}$. The high $\mathrm{q}^{2}$ value is the best indicator of 2D QSAR's reliability since only a high $\mathrm{r}^{2}$ could be due to data overfitting. Quite often, a $\mathrm{q}^{2}$ value of more than 0.5 is considered appropriate. (Golbraikh and Tropsha, 2002; Doweyko, 2004; Ponce et al., 2004).

The developed models showed that fungicidal activity was inversely related to descriptors, DeltaEpsilonC and AI descriptor, T_2_Cl_6, T_2_F_6, and T_T_F_3 and directly related to ZcompDipole. Two descriptors viz. DeltaEpsilonC and T_2_Cl_6 significantly $(\sim 70 \%)$ impact the fungicidal activity of test compounds. Alignment Independent (AI) descriptors were estimated, as explained in Baumann's paper (Balaban, 1982), on the basis of molecular topology, type of bond, and atom. Every atom was given a minimum of one and a maximum of three attributes. Molecular topology (T) was designated as the first attribute, followed by atom symbol and atoms linked with multiple (double or triple) bonds as second and third attribute, respectively. Then, selective distance count statistics, which counts all the fragments between the first atom and the last atom isolated by a graph distance, for all combinations of various attributes were calculated. Graph distance is the least number of atoms across the path joining two atoms in molecular structure. For example, selective distance count statistic
"AB2" (e.g., TOPO2N3) counts all the fragments between a start atom with attribute "A" (e.g., " 2 " a double bonded atom) and an end atom with attribute "B" (e.g., "N") separated by a graph distance 3. Topological indices are numerical values associated with chemical constitutions which establish correlation between biological activity and chemical structure. AI descriptors in this study were calculated with the help of attributes namely, 2 (atom with double bond), 3 (atom with double bond), C (Carbon), N (Nitrogen), O (Oxygen), S (Sulfur), $\mathrm{H}$ (Hydrogen), $\mathrm{F}$ (Fluorine), $\mathrm{Cl}$ (Chlorine), and $\mathrm{Br}$ (Bromine) with distance ranging from 0 to 7 . DeltaEpsilonC is a measure of contribution of electronegativity. The result revealed that it is negatively correlated with fungicidal activity of the test compounds.

\section{CONCLUSION}

The study revealed that all test compounds showed fungicidal activity against $S$. rolfsii., but compound $\mathbf{4 r}$ showed the highest activity. The QSAR study determined quantitative correlation between fungicidal activity and structural/physicochemical properties of test compounds. The variables in developed model equations established that structural, molecular shape analysis, electronic, and thermodynamic descriptors played a major role in fungicidal activity of the compounds. In the case of MLR and PLS, the overall prediction was found to be around 94 and $80 \%$, respectively. The 2D-QSAR study revealed that results of MLR analysis exhibited significant predictive power and reliability than the other two methods (PCR and PLS). Information and understanding of descriptors influencing fungicidal activity of these chromones could be used for structure optimization to improve activity.

\section{DATA AVAILABILITY STATEMENT}

The original contributions presented in the study are included in the article/Supplementary Material; further inquiries can be directed to the corresponding author.

\section{AUTHOR CONTRIBUTIONS}

PK and NS conceptualized the idea; VR did HRMS interpretation.

\section{ACKNOWLEDGMENTS}

The author (PK) is thankful to ICAR-IARI, New Delhi, India for providing all the facilities for research work.

\section{SUPPLEMENTARY MATERIAL}

The Supplementary Material for this article can be found online at: https://www.frontiersin.org/articles/10.3389/fchem.2021.636882/ full\#supplementary-material. 


\section{REFERENCES}

Abbott, W. S. (1925). A method of computing the effectiveness of an insecticide. J. Econ. Entomol. 18, 265-267. doi:10.1093/jee/18.2.265a

Aycock, R. (1966). Stem rot and other diseases caused by Sclerotium rolfsii. Raleigh, NC: North Carolina State University.

Balaban, A. T. (1982). Highly discriminating distance-based topological index. Chem. Phys. Lett. 89, 399-404. doi:10.1016/0009-2614(82)80009-2

Croux, C., and Joossens, K. (2005). Influence of observations on the misclassification probability in quadratic discriminant analysis. J. Multivar. Anal. 96, 384-403. doi:10.1016/j.jmva.2004.11.001

Deising, H. B., Reimann, S., Peil, A., and Weber, W. E. (2002). "Disease management of rusts and powdery mildews," in The mycota XI. Application in agriculture. Editor F. Kempken (Berlin, Germany: Springer), 243-269.

Devillers, J. (1996). Neuronal network in QSAR and drug design. London, United Kingdom: Academic Press.

Doweyko, A. M. (2004). 3D-QSAR illusions. J. Comput. Aided Mol. Des. 18, 587-596. doi:10.1007/s10822-004-4068-0

Gammill, R. B. (1979). A new and efficient synthesis of 3-halogenated $4 \mathrm{H}-$ 1-Benzopyran-4-ones. Synthesis 1979, 901-903. doi:10.1055/s-197928869

Gaspar, A., Matos, M. J., Garrido, J., Uriarte, E., and Borges, F. (2014). Chromone: a valid scaffold in medicinal chemistry. Chem. Rev. 114, 4960-4992. doi:10.1021/ cr400265z

Gaspar, A., Silva, T., Yáñez, M., Vina, D., Orallo, F., Ortuso, F., et al. (2011). Chromone, a privileged scaffold for the development of monoamine oxidase inhibitors. J. Med. Chem. 54, 5165-5173. doi:10.1021/jm2004267

Gasparová, R., Lácová, M., El-Shaaer, H. M., and Odlerová, Z. (1997). Synthesis and antimycobacterial activity of some new 3-heterocyclic substituted chromones. Farmaco 52, 251-253.

Golbraikh, A., and Tropsha, A. (2002). Beware of $\mathrm{q}^{2}$ !. J. Mol. Graph. Model. 20, 269-276. doi:10.1016/s1093-3263(01)00123-1

Hollomon, D. W. (2016). Fungicide resistance: facing the challenge-a review. Plant Protect. Sci. 51, 170-176. doi:10.17221/42/2015-PPS

Huberty, C. J. (1994). Applied discriminant analysis. New York, NY: Willey.
Hudson, B. D., Hyde, R. M., Rahr, E., Wood, J., and Osman, J. (1996). Parameter based methods for compound selection from chemical databases. Quant. Struct. Act. Relat. 15, 285-289. doi:10.1002/qsar.19960150402

Kaushik, P., Sarkar, D. J., Chander, S., Rana, V. S., and Shakil, N. A. (2019). Insecticidal activity of phenolic acid amides against brown planthopper $(\mathrm{BPH})$, Nilaparvata lugens (Stål) and their QSAR analysis. J. Environ. Sci. Health B 54, 489-497. doi:10.1080/03601234.2019.1574174

Kubyani, H. (1994). Variable selection in QSAR studies: an evolutionary algorithm. Quant. Struct. Act. Relat. 13, 285-294. doi:10.1002/qsar.19940130306

Nene, Y. L., and Thapliyal, P. N. (1979). in "Fungicides in plant disease control," in Evaluation of fungicides. Editors Y. L. Nene and P. N. Thapliyal (New Delhi, India: Oxford and IBH Publishing Co.), 406-428.

Oerke, E.-C. (2006). Crop losses to pests. J. Agric. Sci. 144, 31-43. doi:10.1017/ s0021859605005708

Ponce, Y. M., Garit, J. A. C., Torrens, F., Zaldivar, V. R., and Castro, E. A. (2004). Atom, atom-type, and total linear indices of the "molecular pseudograph's atom adjacency matrix": application to QSPR/QSAR studies of organic compounds. Molecules 9, 1100-1123. doi:10.3390/91201100

Punja, Z. K. (1985). The biology, ecology, and control of Sclerotium rolfsh. Annu. Rev. Phytopathol. 23, 97-127. doi:10.1146/annurev.py.23.090185.000525

Yadav, D. K., Kaushik, P., Pankaj, V. S., Rana, D., Khatri, D., et al. (2019). Microwave assisted synthesis, characterization and biological activities of ferrocenyl chalcones and their QSAR analysis. Front. Chem. 7, 814. doi:10. 3389/fchem.2019.00814

Conflict of Interest: The authors declare that the research was conducted in the absence of any commercial or financial relationships that could be construed as a potential conflict of interest.

Copyright (c) 2021 Kaushik, Shakil and Rana. This is an open-access article distributed under the terms of the Creative Commons Attribution License (CC $B Y)$. The use, distribution or reproduction in other forums is permitted, provided the original author(s) and the copyright owner(s) are credited and that the original publication in this journal is cited, in accordance with accepted academic practice. No use, distribution or reproduction is permitted which does not comply with these terms. 